\title{
Refilling process in the plasmasphere: a 3-D statistical characterization based on Cluster density observations
}

\author{
G. Lointier ${ }^{1}$, F. Darrouzet ${ }^{2}$, P. M. E. Décréau ${ }^{1}$, X. Vallières $^{1}$, S. Kougblénou ${ }^{1}$, J. G. Trotignon ${ }^{1}$, and J.-L. Rauch ${ }^{1}$ \\ ${ }^{1}$ Laboratoire de Physique et Chimie de l'Environnement et de l'Espace, UMR 7328 CNRS/University of Orléans, 3A avenue \\ de la recherche Scientifique, 45071 Orléans cedex 2, France \\ ${ }^{2}$ Belgian Institute for Space Aeronomy (IASB-BIRA), Brussels, Belgium
}

Correspondence to: G. Lointier (guillaume.lointier@cnrs-orleans.fr)

Received: 30 June 2011 - Revised: 1 October 2012 - Accepted: 2 October 2012 - Published: 8 February 2013

\begin{abstract}
The Cluster mission offers an excellent opportunity to investigate the evolution of the plasma population in a large part of the inner magnetosphere, explored near its orbit's perigee, over a complete solar cycle. The WHISPER sounder, on board each satellite of the mission, is particularly suitable to study the electron density in this region, between 0.2 and $80 \mathrm{~cm}^{-3}$. Compiling WHISPER observations during 1339 perigee passes distributed over more than three years of the Cluster mission, we present first results of a statistical analysis dedicated to the study of the electron density morphology and dynamics along and across magnetic field lines between $L=2$ and $L=10$. In this study, we examine a specific topic: the refilling of the plasmasphere and trough regions during extended periods of quiet magnetic conditions. To do so, we survey the evolution of the ap index during the days preceding each perigee crossing and sort out electron density profiles along the orbit according to three classes, namely after respectively less than 2 days, between 2 and 4 days, and more than 4 days of quiet magnetic conditions (ap $\leq 15 \mathrm{nT}$ ) following an active episode (ap $>15 \mathrm{nT}$ ). This leads to three independent data subsets. Comparisons between density distributions in the 3-D plasmasphere and trough regions at the three stages of quiet magnetosphere provide novel views about the distribution of matter inside the inner magnetosphere during several days of low activity. Clear signatures of a refilling process inside an expended plasmasphere in formation are noted. A plasmapause-like boundary, at $L \sim 6$ for all MLT sectors, is formed after 3 to 4 days and expends somewhat further after that. In the outer part of the plasmasphere $(L \sim 8)$, latitudinal profiles of median density values vary essentially according to the MLT sector considered rather than according to the refilling dura-
\end{abstract}

tion. The shape of these density profiles indicates that magnetic flux tubes are not fully replenished after 6 days of quiet conditions. In addition, the outer plasmasphere in the night and dawn sectors (22:00 to 10:00 MLT range) maintains an overall clear deficit of ionospheric population, when compared to the situation in the noon and dusk sectors (10:00 to 22:00 MLT range).

Keywords. Magnetospheric physics (Plasmasphere)

\section{Introduction}

The plasmasphere consists of a cold $(\leq 1 \mathrm{eV})$ and dense (10$10^{4} \mathrm{~cm}^{-3}$ ) torus of plasma that co-rotates with the Earth, forming the innermost region of the magnetosphere (Carpenter, 1963; Nishida, 1966; Lemaire and Gringauz, 1998). Its outer edge, the plasmapause, has been recently referred to as the Plasmasphere Boundary Layer, or PBL, because of the complexity and variability of the plasma population that can exist near this boundary and because of the variety of criteria used to define it (Carpenter and Lemaire, 2004). It is widely recognised that the plasmasphere is a very dynamic system highly constrained by both the solar wind and the ionosphere via the solar wind driven convection electric field, the electric field associated with the Earth's rotation and the interchange fluxes between the ionosphere and magnetospheric regions at higher altitude. Variations of these large-scale processes and their interplay affect the configuration and the dynamics of the plasmasphere, i.e. have an impact on the plasma distribution in space and time and govern the PBL formation (Darrouzet et al., 2009a). 
The arrival of a solar wind perturbation at Earth induces an increase of the convection electric field. The convecting flux tubes outside the co-rotation region drive their plasma content sunward toward the magnetopause (McFadden et al., 2008) before being ultimately driven tailward (Chandler and Moore, 2003; Elphic et al., 1997). During disturbed geomagnetic periods, the convection electric field is strong enough to erode outer regions of the plasmasphere. The most spectacular feature of the plasmasphere dynamics under a geomagnetic activity increase is the formation of drainage plumes (elongated structures of cold plasma) in the afternoon local time sector (Goldstein et al., 2004; Darrouzet et al., 2008). The density transition at the PBL gets sharper and moves earthward, below $L=3$, on timescale of a few hours (Carpenter, 1967; Chappell et al., 1970; Carpenter and Anderson, 1992; Moldwin et al., 2002). Additional processes such as the subauroral ion drift and various wave activities can affect the spatial distribution of the density at the PBL (De Keyser et al., 1998; Moldwin et al., 1997).

The refilling process of the plasmasphere, continually supplied by escaping particles from the dayside ionosphere along the co-rotating flux tubes, is more effective during extended periods of low geomagnetic activity $\left(K_{p}<1+\right.$, i.e. ap $<5 \mathrm{nT}$ ) (Darrouzet et al., 2008). Two or three days of refilling are typically required for plasma density to reach high values at geosynchronous orbit and beyond, but this time interval is not sufficient for diffusive equilibrium with the ionosphere to be established (Moldwin et al., 1994; Spasojević et al., 2003). In contrast, Reinisch et al. (2004) reported a significant filling in less than $28 \mathrm{~h}$ at lower altitudes $R=2.5 R_{\mathrm{E}}$. The unexpected long refilling time of flux tubes in the outer plasmasphere implies that a transport mechanism, in addition to co-rotation and convection, drifts the plasma outward across the geomagnetic field lines. The existence of a plasmaspheric wind mechanism was first predicted by Lemaire and Schunk (1992), and observed later by Dandouras (2008).

The description of the plasmasphere's dynamics assumes that the PBL is always observable due to the continuous interplay between the convection electric field and the corotation one. In that line of thought, several studies have been achieved to elaborate an empirical relationship between the PBL location and the geomagnetic indices (Carpenter and Anderson, 1992; Moldwin et al., 2002), and between the PBL location and the solar wind conditions (Larsen et al., 2007). However, observations of smooth plasmaspheric density transition extended to $L=7$ and beyond have been reported after several days of quiet geomagnetic conditions (Chappell, 1972; Carpenter and Anderson, 1992; Tu et al., 2006). This implies either a PBL located beyond $L=7$ or a smooth density transition from the inner plasmasphere up to the sub-auroral region without detectable signature of a distinct boundary. Such a smooth transition is possible if the co-rotation dominates over the convection at large radial distance. Tu et al. (2007) presented some cases of extended plasmasphere having smooth density transition for weak geo- magnetic activity $\left(K_{p}<3\right)$ during two or more days. They suggest that this is indirect observational evidence of the plasmaspheric wind. Little attention has been paid to model such a smooth density transition at the PBL. Actually, ignoring the density distribution along depleted flux tubes is a real difficulty when one wishes to undertake and validate simulations of plasmasphere filling/refilling (Tu et al., 2004, 2006, 2007).

Wave and particle interactions impact the density distribution inside the plasmasphere (Liemohn et al., 1997). Conversely, knowledge of the electron density $n_{\mathrm{e}}$ in the plasmasphere and its outer edge is necessary to study how dense cold plasma can affect whistler-mode waves that scatter radiation belt electrons $(3<L<7)$. Many studies demonstrated that the presence of ElectroMagnetic Ion-Cyclotron (EMIC) waves (Summers and Thorne, 2003; Spasojević et al., 2004; Borovsky and Steinberg, 2006; Usanova et al., 2010), plasmaspheric hiss (Lam et al., 2007) and whistler-mode chorus (Summers et al., 2004) at the PBL and within the drainage plumes can pitch-angle scatter relativistic electrons during storms (at different time scales, depending on the waves). Borovsky and Denton (2009) show clear evidence that buildup of the outer plasmasphere plays an important role in the decay of the radiation belt electrons $(\sim 150 \mathrm{keV})$ during intervals of extreme geomagnetic calm. Ring current protons may also be lost due to EMIC waves (Spasojević et al., 2004). It is necessary to model the density distribution along the magnetic field lines in order to understand the growth of these waves and their impact on the plasma populations.

Most thermal plasma density models are empirical. The model of Carpenter and Anderson (1992) describes the equatorial electron density based on the near equatorial ISEE-1 radio measurements. The IZMIRAN model by Chasovitin et al. (1998) and the Global Core Plasma Model (GCPM) by Gallagher et al. (2000) yield vertical profiles of plasmaspheric density as a function of solar and geomagnetic activities. Sheeley et al. (2001) used CRRES observations to describe the density inside and outside the plasmasphere. In these models, an electron density profile of $L^{\alpha}$ (with $-4.15 \leq \alpha \leq-3.45$ ) describes reasonably well the density distribution in the equatorial plane over a $L$-shell range of $1.6<L<7$ (Reinisch et al., 2009). In contrast, the latitudinal dependence of $n_{\mathrm{e}}$ along field lines is less well known. In order to infer the electron density profile along an individual field line from in situ observations of POLAR and CLUSTER spacecraft, Denton et al. (2002a,b, 2004, 2009) assumed a power law dependence with respect to the geocentric radius, $n_{\mathrm{e}}=n_{\mathrm{e} 0}\left(L R_{\mathrm{E}} / R\right)^{p}$, where $R$ is the geocentric radius, $L R_{\mathrm{E}}$ is the geocentric radius $R$ at the apex, $n_{\mathrm{e} 0}$ is the equatorial electron density and $p$ is the power law parameter. This method assumes that the equatorial density distribution is known (from another model or from in situ measurements), which is a real difficulty, near the PBL region. Moreover, this method works only when the POLAR 
trajectory crosses a particular field line at two different points (one in each hemisphere). Tu et al. (2006) extended the technique used by Reinisch et al. $(2001,2004)$ and Huang et al. (2004) to model the field-aligned electron density profile remotely from the Radio Plasma Imager (RPI) on board the IMAGE satellite. They concluded that the power law dependence quoted above is reliable enough to construct global empirical plasmasphere/trough models. They discussed the feasibility of determining the density profile along depleted flux tubes but they could not obtain $n_{\mathrm{e} 0}$ because measurements covered only one hemisphere at a time. These studies reveal all the inherent difficulty in reconstructing density profiles along magnetic field line from direct in situ and remote data. Nevertheless, the density variations across the fields lines are more pronounced than those along the field lines (Darrouzet et al., 2006) and the parallel velocity of the electron is almost constant among the field lines (Tu et al., 2005). This suggests that a statistical approach can be suitable to study field-aligned density distribution in the plasmasphere region.

Based on a statistical description of the WHISPER observations taken on board the Cluster satellites, this paper focuses on the latitudinal dependence of the electron density along the magnetic flux tubes and its evolution during extended intervals of low magnetic activity. After a presentation of the instrumentation and of the method used to elaborate the database (Sect. 2), statistical results are discussed in Sect. 3. The last section summarises our findings and discusses their perspectives.

\section{Cluster plasmaspheric coverage}

The ESA's Cluster mission, launched in 2000, is a constellation of four identical spacecraft (referred to here as $\mathrm{C} 1$, $\mathrm{C} 2, \mathrm{C} 3$ and $\mathrm{C} 4$ ) flying in a tetrahedral configuration (Escoubet et al., 1997). The Cluster mission has been designed to study the small-scale structures and macroscopic turbulence in three dimensions in many regions of the magnetosphere (magnetopause, polar cusps, magnetotail and the auroral zones) as well as the solar wind upstream of the bow shock (Escoubet et al., 2001). The four spacecraft have originally evolved along a quasi-polar orbit, with a perigee at $4.2 R_{\mathrm{E}}$, an apogee at $19.6 R_{\mathrm{E}}$ and a periodicity of about $57 \mathrm{~h}$. Cluster orbit precession covers a local time sector of about $0.15 \mathrm{~h}$ between two perigee passes, enabling spacecraft to cross the aforementioned regions at all local time sectors in one year. During the nominal part of the mission, the interspacecraft distance was changed several times, ranging from $100 \mathrm{~km}$ to $10000 \mathrm{~km}$. Since 2005 , the constellation has been altered from a tetrahedral configuration to a multi-scale geometry; C1, C2 and C3 kept the same separation distance while $\mathrm{C} 4$ orbited at closer distance to $\mathrm{C} 3$ (down to $17 \mathrm{~km}$ ). The plasmasphere region, which is the region of interest for this study, was not one of the primary scientific objectives.
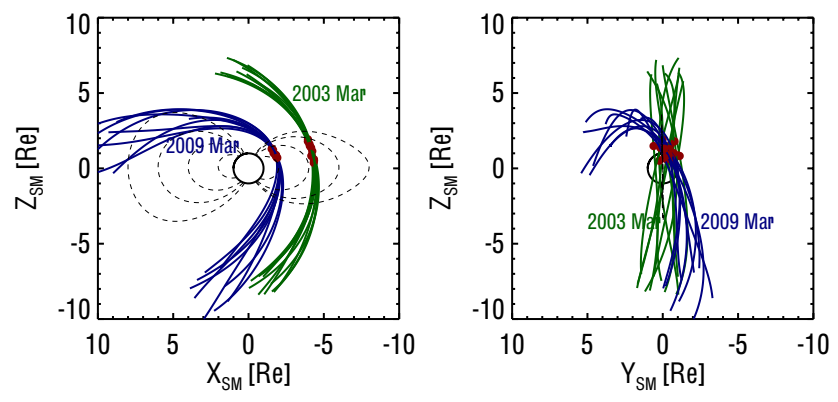

Fig. 1. Example of successive perigee passes by C2 for March 2003 (green) and March 2009 (blue) projected in SM coordinates. Dashed black lines draw magnetic field lines estimated by the geomagnetic model IGRF-11 + Tsy04. Red dots are placed at the perigees of the plotted orbits.

However, the high eccentricity of the Cluster's orbit enables spacecraft to dive deeply enough into the inner magnetosphere to cross the plasmaspheric region. During the first years of the mission, a large range of latitudes over a limited local time interval is explored at each perigee crossing. The PBL is typically located in the $3<L<8$ range at the equator, depending on the level of geomagnetic activity (Carpenter and Lemaire, 2004). Darrouzet et al. (2009a) reviewed the contribution of the Cluster mission in our understanding of the plasmaspheric structures and dynamics and also its contribution to recent advances in the description by models.

Cluster orbit has naturally and slowly evolved over the course of the mission. Figure 1 shows a few $\mathrm{C} 2$ orbit elements around consecutive perigee passes in March 2003 (green curves), and in March 2009 (blue curves). Red dots identify perigee positions of the selected passes. Dashed lines indicate magnetic field lines computed from the IGRF-11 (Finlay et al., 2010) and Tsyganenko 2004 models (Tsyganenko and Sitnov, 2005) under the geomagnetic conditions defined by default for this model, presented hereafter in Sect. 2.3. The figure displays orbit projections onto the $\mathrm{X}-\mathrm{Z}$ and $\mathrm{Y}-\mathrm{Z}$ planes in solar magnetic (SM) coordinates. It illustrates clearly the slow drift to lower altitudes of the perigee passes. At the beginning of the Cluster mission, the perigee was close to the ecliptic plane and, due to a high orbit inclination angle, plasmasphere crossings from the Southern to the Northern Hemisphere were relatively symmetric with respect to the magnetic equator plane (see the SM Y$\mathrm{Z}$ plane). As years go by, the inclination angle decreases; the drift of the orbit results in an asymmetric plasmasphere crossing. The spacecraft do not enter and leave the plasmasphere at similar MLT values but sweep a significant range of MLT values. Moreover, the latitudinal range explored during the crossing is smaller than at the start of the mission (see also Kougblénou et al., 2011, for a discussion about the Cluster orbit evolution). As the perigee geocentric distance decreases (from $4.2 R_{\mathrm{E}}$ at launch time to $1.5 R_{\mathrm{E}}$ in 2010), similar Z-values of perigee lead to higher latitudes 
in 2010 (above $25^{\circ} \mathrm{N}$ ). The perigee, which explored regions near apex of magnetic field lines in 2003, explored regions closer to their magnetic foot in 2010. It is important to note that from the middle of the year 2008, the Cluster orbit enters twice the plasmasphere. The spacecraft cross the inner plasmasphere from South to North in a given local time sector, heading toward perigee, then fly over auroral regions and polar cap at higher altitudes. Finally, the spacecraft enter again in plasmaspheric magnetic flux tubes located at $6<L<9$ in a local time sector opposite to that of the inbound crossing, travelling from the Northern to the Southern Hemisphere at a slower velocity (during a longer time interval) than inbound. During the years selected for this analysis, the satellites generally leave the plasmasphere during the second crossing before reaching the equator. We discuss in Sect. 2.2 the specifics of electron density sampling during the second plasmasphere crossing. Independently of the number of times the orbit enters and leaves the plasmasphere or trough regions near a given perigee, we define a density profile as constructed from all density records measured along that perigee pass by a given Cluster satellite.

Figure 1 illustrates that, in addition to a slow evolution over the years, the spacecraft cross the magnetic field lines at different locations from one orbit to another. This is a consequence of the Earth rotation coupled with the magnetic dipole tilt. Projected onto the X-Z and X-Y planes in SM coordinates, the position of the perigee (red dot) varies greatly in latitude and in local time. This variability is enhanced under the effect of geomagnetic activity variations and gives access to a larger range of latitudes along magnetic field lines.

\subsection{Electron density determination}

We use data obtained from one of the eleven Cluster instruments, called Wave of HIgh frequency and Sounder for Probing Electron density by Relaxation (WHISPER) to estimate the electron density (Décréau et al., 1997). Particle instruments on board Cluster spacecraft cannot be used to estimate the electron density in this region. The PEACE instrument (Johnstone et al., 1997), measuring electrons in the low energy range $(10 \mathrm{eV}-26.5 \mathrm{keV})$, is switched off most of the time near the perigee due to the presence of high energetic populations in the inner magnetosphere (mainly in the radiation belts and lesser in the ring current population), which can be hazardous for particle measurements. WHISPER is a relaxation sounder providing a diagnosis of the local particle populations from triggering resonances in the plasma in active (sounding) mode and from monitoring the natural electric waves in passive mode (Décréau et al., 1997, 2001).

In this work, we present electron density estimates obtained after an analysis of frequency spectra obtained during both operation modes (sounding or passive). This analysis carries out a direct or indirect identification of the electron plasma frequency $F_{\text {pe }}$, related to the electron density $n_{\mathrm{e}}$ as $F_{\mathrm{pe}}[\mathrm{kHz}] \approx 9 \sqrt{n_{\mathrm{e}}}\left[\mathrm{cm}^{-3}\right]$. The WHISPER instrument

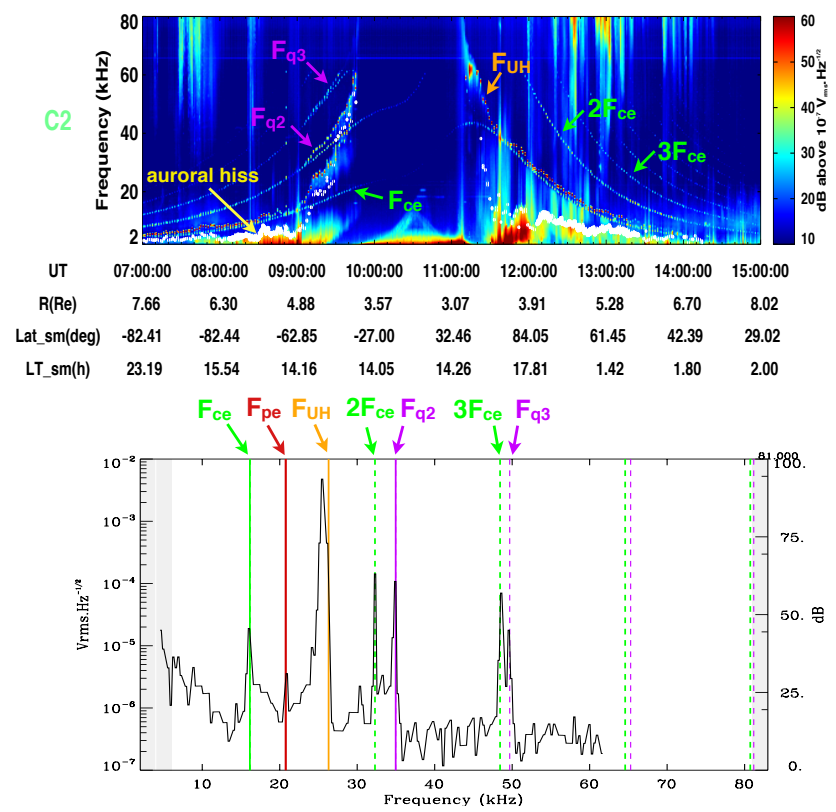

Fig. 2. Example of an asymmetric plasmasphere crossing, on $9 \mathrm{Au}-$ gust 2007, 07:00-15:00 UT. Top panel shows the spectrogram recorded by $\mathrm{C} 2$. Bottom panel displays a spectrum recorded at 09:16:10 UT. Typical resonances observed in the plasmasphere are indicated on both panels by arrow of given colours: orange for upper hybrid frequency $\left(F_{\mathrm{UH}}\right)$, green for gyro-harmonic $\left(n F_{\mathrm{ce}}\right)$ and purple for Bernstein modes $\left(F_{\mathrm{qn}}\right)$. The plasma frequency $F_{\mathrm{pe}}$ derived from the diagnostic is plotted as white dots in the top panel (it is an identified resonance pointed by a red arrow in the bottom panel). Auroral hiss emissions (top panel) are pointed by a yellow arrow.

can thus estimate electron densities for values up to $80 \mathrm{~cm}^{-3}$ with a temporal resolution of $2 \mathrm{~s}$ on average. The estimated spatial resolution of electron density observed is of the order of $1 \mathrm{~km}$, i.e. a few times the electric antenna tip-to-tip length $d$, with $d=88 \mathrm{~m}$. The electron density determination has been carried out in two steps: (1) a semi-automatic tool dedicated to the density production for the Cluster Active Archive (Trotignon et al., 2010) has been first applied; (2) the obtained electron density profiles are then checked manually in order to correct or filter spurious points.

In the plasmasphere, resonances arise at the electron gyrofrequency $F_{\mathrm{ce}}$ (and its harmonics $n F_{\mathrm{ce}}$ ), the total electron plasma frequency $F_{\mathrm{pe}}$, the upper-hybrid frequency $F_{\mathrm{UH}}$ and the Bernstein's mode frequencies $F_{\mathrm{qn}}$ (Bernstein, 1958). Note that local wave cut-off properties can also be used to determine $F_{\text {pe }}$ (Canu et al., 2001). We can further mention the lower hybrid frequency, $F_{\mathrm{lh}}$, observed since 2009 at altitudes below $\sim 4000 \mathrm{~km}$ (Kougblénou et al., 2011).

The top panel of Fig. 2 shows a spectrogram recorded during a plasmasphere crossing by $\mathrm{C} 2$ on 9 August 2007 between 07:00 and 15:00 UT. This spectrogram, which combines both active and passive measurements, displays typical 
resonances allowing electron density to be accurately determined (Trotignon et al., 1986, 2010). White dots point out the $F_{\text {pe }}$ profile along the orbit. For an active spectrum, the $F_{\text {pe }}$ value is deduced semi-automatically from the diagnostic of $F_{\mathrm{UH}}$ and $n F_{\mathrm{ce}}$ series, which are related together by the relation $F_{\mathrm{UH}}=\sqrt{F_{\mathrm{pe}}^{2}+F_{\mathrm{ce}}^{2}} \cdot F_{\mathrm{UH}}$ is assumed to be the most intense resonance in a frequency band manually selected (Trotignon et al., 2010). $F_{\mathrm{ce}}$ is derived from the magnetic field measurement provided by FGM (Balogh et al., 1997) $\left(F_{\mathrm{ce}}[\mathrm{kHz}]=28.10^{-3} \times B_{0}[\mathrm{nT}]\right)$. The high frequency limit of the instrument, $80 \mathrm{kHz}$, is also the highest observable value of $F_{\mathrm{UH}}$. The highest plasma frequency measurable, $F_{\text {pe_max }}$ around perigee, is controlled by the gyrofrequency value, and is smaller than $80 \mathrm{kHz}$. $F_{\text {pe_max }}$ is always close to $80 \mathrm{kHz}$ during the first part of the mission when perigee is at $4 R_{\mathrm{E}}$ and the gyrofrequency $F_{\text {ce }}$ below $\sim 20 \mathrm{kHz}\left(F_{\text {pe_max }}=77 \mathrm{kHz}\right.$ when $F_{\mathrm{ce}}=20 \mathrm{kHz}$ ). Later in the mission phase, the gyrofrequency at perigee increases with decreasing radial distances and increasing latitudes. For instance, the gyrofrequency value is $F_{\mathrm{ce}} \sim 40 \mathrm{kHz}$ at a magnetic latitude of $50^{\circ}$ and a radial distance of $3 R_{\mathrm{E}}$, which leads to $F_{\mathrm{pe} \max }=70 \mathrm{kHz}$ and hence to a highest measurable density $n_{\mathrm{e}}=60 \mathrm{~cm}^{-3}$. Another difficulty occurs when $F_{\mathrm{UH}}$ is close to $F_{\mathrm{ce}}$. In this case the $163 \mathrm{~Hz}$ instrumental frequency resolution limits the accuracy on $F_{\text {pe }}$ measurements. When this happens at high latitudes, auroral hiss emissions are often present, displaying an upper frequency cutoff assumed to occur at the local plasma frequency (Persoon et al., 1988). The upper frequency cutoff signatures, identified semi-automatically in the passive spectrograms, then enable the $F_{\text {pe }}$ profile to be completed. This situation is encountered before $\sim 09: 00$ UT and after $\sim$ 12:00 UT in Fig. 2.

The obtained electron density profiles are then checked, orbit by orbit, in order to validate them. We remove spurious $F_{\text {pe }}$ values after visual inspection by overlaying the determined $F_{\text {pe }}$ profile on the matching spectrogram. Noting that, when $F_{\text {pe }}$ values are obtained in sounding mode, it is possible to correct a wrong diagnostic by taking into account all triggered resonances. Indeed, under the approximation of a Maxwellian plasma (Belmont, 1981), the relative position of the measured resonances, including Bernstein resonances $F_{\mathrm{qn}}$, allows $F_{\mathrm{pe}}$ estimation to be confirmed. The bottom panel of the Fig. 2 shows an example of an active spectrum recorded at 09:16:10 UT on board C2. Vertical lines indicate the identified resonances. More precisely, the frequency at $F_{\mathrm{UH}}$ resonance leads to a first calculated value of $F_{\mathrm{pe}}$, where it is possible to check the presence of another resonance. A second independent value of $F_{\mathrm{pe}}$ can be derived from the $F_{\mathrm{qn}}$ frequencies. In the chosen example, it is placed so close to the first $F_{\text {pe }}$ estimate that it has not been shown in Fig. 2. This approach is particularly useful when the signalto-noise ratio at $F_{\mathrm{pe}}$ and $F_{\mathrm{UH}}$ resonances is low, noting that it is typically higher at Bernstein resonances. At the end of the validation process, we get complete and reliable electron density profiles including either $n_{\mathrm{e}}$ estimations in the range 0.2 to $80 \mathrm{~cm}^{-3}$ or records with the qualitative information that the density is high $\left(F_{\mathrm{UH}}\right.$ above $80 \mathrm{kHz}$ and significantly above $F_{\text {ce }}$ ).

\subsection{Dataset selection}

We derive electron density profiles around perigees of $\mathrm{C} 1$, C2, C3 and C4 during two distinct periods from 1 June 2002 to 1 June 2003, and from 1 January 2007 to 30 April 2009. These two periods enable us to study the two types of orbits described above (see Sect. 2) and thus to explore different regions of the inner magnetosphere. The first period is associated with the maximum of solar activity during solar cycle 23 . The second one is associated with the minimum of solar activity between solar cycle 23 and 24 . This corresponds to two distinct regimes of disturbance level in the magnetosphere. All geomagnetic local time sectors are covered in each of the two periods. The two periods have also been selected because of the large inter-spacecraft distances $\left(\gtrsim 1 R_{\mathrm{E}}\right)$. This limits possible spatial redundancies in the dataset. Statistics gather 464 perigee passes with data acquisition from the WHISPER instrument.

The orbit element analysed around the perigee is not limited to the plasmasphere region, which is often an elusive entity. The density transition at the PBL is not always observed (Tu et al., 2007; Darrouzet et al., 2009b). Functional forms developed in the past infer the PBL location at the equator only and have the disadvantage of not taking into account the individual history of the plasmasphere (Carpenter and Anderson, 1992; Larsen et al., 2007). Moreover, we needed to explore the outer plasmasphere, or trough region, as well as the plasmasphere itself. We decided thus to follow each electron density profile over a large region on both sides of the perigee. We stop the analysis whenever the electron density decreases below the $0.2 \mathrm{~cm}^{-3}$ limit (i.e. under the WHISPER instrument lower threshold) for a significant time interval, or when spacecraft cross another magnetosphere region easily recognisable from the WHISPER data (e.g. cusp, magnetosheath, etc.). In any event, we limit our dataset to regions where the Macllwain parameter $L$ is below 10 . Within this limit, regions at $L>8$ consist of a relatively tenuous plasma and the lowest densities $\left(<0.2 \mathrm{~cm}^{-3}\right)$, for instance inside holes, are ignored, raising a reliability issue about the density profile sampled. This happens in particular when spacecraft move on the fringes of the plasmasphere during several hours, outbound from perigee (and far from auroral hiss emissions), after year 2008. This situation has to be taken into account when discussing global quantities derived from statistics, as it leads to over-estimated mean or median electron density values.

Every density profile is then re-sampled, averaging all density measured (from passive or active diagnostics) in intervals of one minute. The distance covered in one minute by Cluster spacecraft depends on their latitude and on the 

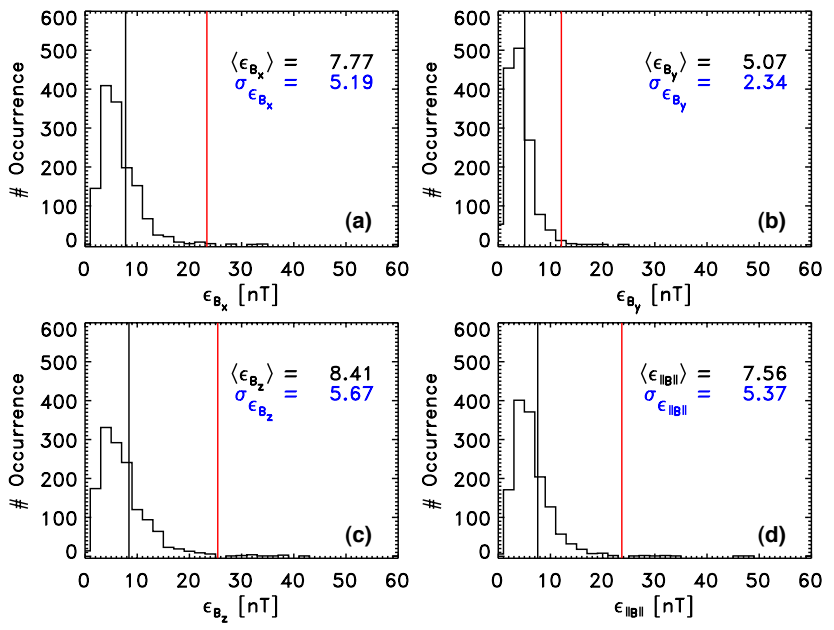

Fig. 3. Comparison of magnetic field vectors values estimated by the geomagnetic model IGRF-11 + Tsy04 with values measured by the FGM instrument. Panels (a), (b), (c) and (d) refer to the $x, y, z$ vector components and overall magnitude. Black vertical lines depict, respectively, the mean value of distributions displayed in each panel. Red vertical lines set the limit of rejected cases.

orbit configuration. It varies from year 2002, when the distance is $\sim 300 \mathrm{~km}$ three hours before perigee and $\sim 250 \mathrm{~km}$ three hours after perigee, to year 2008, when the distances three hours before and after perigee amount to, respectively, $\sim 400 \mathrm{~km}$ and $\sim 200 \mathrm{~km}$.

\subsection{Magnetic field coordinate system}

To study how the electron density distribution is organised by the geomagnetic field lines, positions of electron density records are transformed into a geomagnetic coordinate system. From each position of observation, $M$, we follow the magnetic field line up to its apex, $A$, which defines the parameter $L$ (i.e. the geocentric distance of $A$, expressed in Earth-radii). MLat is the angle between geocentric vectors at apex $A$, and at position $M$. MLT is defined by the magnetic longitude at apex $A$. To do that, we need a robust geomagnetic model able to reproduce accurately all the variability encompassed in the dataset. By comparing results of several geomagnetic models and FGM magnetic measurements, we chose to combine the IGRF-11 internal geomagnetic model (Finlay et al., 2010) with the Tsyganenko's external geomagnetic model version 2004, Tsy04 (Tsyganenko and Sitnov, 2005). These models have the advantage of being constrained by a combination of geo-effective instantaneous parameters (solar wind density and speed, magnitude of the $B_{\mathrm{y}}$ and $B_{\mathrm{z}}$ components of the interplanetary magnetic field and Dst index). By default, the values of these parameters correspond to average interplanetary and ring current conditions. Moreover, Tsy04 considers the cumulative driving of external sources on the magnetosphere, which allows reproducing a rapid evolution of the geomagnetic topology
Table 1. Contribution of each spacecraft to statistics, according to analysed years.

\begin{tabular}{lcccc}
\hline Periods & C1 & C2 & C3 & C4 \\
\hline June 2002 to June 2003 & 121 & 131 & 120 & 0 \\
January 2007 to December 2007 & 140 & 134 & 134 & 136 \\
January 2008 to December 2008 & 110 & 129 & 99 & 0 \\
January 2009 to April 2009 & 20 & 35 & 30 & 0 \\
\hline
\end{tabular}

during unsteady conditions (an evolution ignored in the default version of the model). This is an added value for the statistical analysis presented in this paper. It provides the geomagnetic coordinates of Cluster spacecraft at each element of the electron density profiles according to a realistic magnetic topology and dynamics of the inner magnetosphere at the time resolution of measurements (about $1 \mathrm{~min}$ ).

We retain density profiles for which the model reproduces accurately the FGM measured magnetic field. In order to do so, we calculate the mean absolute deviation $\left(\epsilon_{i}\right)$ between modelled and measured values, over the magnetic field samples attached to the $i$-th density profile of our dataset $\left(\epsilon_{i}=\frac{1}{N} \sum_{j=1}^{N}\left\|\boldsymbol{B}_{j}{ }^{\mathrm{FGM}}-\boldsymbol{B}_{j}{ }^{\mathrm{MOD}}\right\|\right.$, where $j \in[1, \ldots, N]$, $N$ is the number of bins of $i$-th profile). This is calculated, respectively, for the $x, y, z$ vector components of magnetic field and for its magnitude. Figure 3 shows the distribution of the mean absolute deviations $\left(\epsilon_{i}\right)$. Black vertical lines indicate the average of the mean absolute deviations $\langle\epsilon\rangle$. Whatever the magnetic field component, $\langle\epsilon\rangle$ does not exceed $8.5 \mathrm{nT}$, which is about $1 \%$ of the total magnetic field strength variation recorded along a perigee pass. Tsy04 seems to improve slightly the accuracy of description in comparison with previous versions of Tsyganenko's model, like Tsy01, under quiet geomagnetic conditions (Woodfield et al., 2007; Zhang et al., 2010). Note also that, for each component (and for the magnitude), the standard deviation $(\sigma)$ of the mean absolute deviation is of the same order as the average value. This means that any component of the modelled magnetic field is statistically consistent with the measures. Despite the good accuracy of the model, some perigee crossings stand out as outliers. We reject them using the three sigma rule: if for one of the components $\epsilon_{i} \geq\langle\epsilon\rangle+3 \sigma$, we reject the $i$-th profile (see vertical red lines in Fig. 3). This is an objective criterion to discriminate perigee crossings for which geomagnetic conditions are not well enough reproduced.

From the 1387 electron density profiles analysed initially (i.e. when WHISPER data were available), this selection rejected 48 cases, which represents about $3 \%$ of the dataset. Table 1 resumes the final contribution of each spacecraft to statistics. Note that C4's contribution is limited to year 2007 only. For the other years, $\mathrm{C} 4$ is considered to be too close to $\mathrm{C} 3$ for the corresponding measurements to be included in the dataset. Figure $4 \mathrm{a}$ shows the number of electron density measurements contained in our statistics, versus MLT and $L$. 


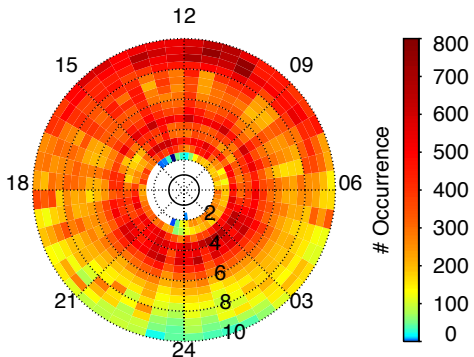

(a)

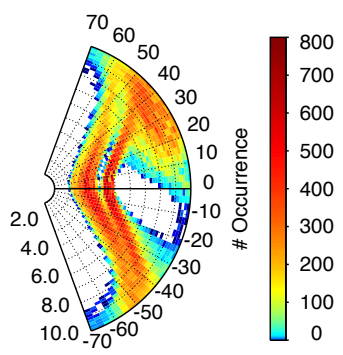

(b)
Fig. 4. Distribution of the electron density measurements gathered after analysis of 1373 validated perigee passes. (a) $L$-MLT view of the occurrence summed up inside magnetic flux tubes of section 0.5 in $L$ and $0.5 \mathrm{~h}$ in MLT. (b) $L$-MLat view of the occurrence summed up over all MLT sectors, for cells of dimension 0.2 in $L$ over the range 2 to 10 and $2.8^{\circ}$ in MLat over the range $-70^{\circ}$ to $70^{\circ}$.

The spatial distribution is almost uniform for $L$ in the $[3,6]$ interval, quasi uniform in the $[6,8]$ interval, and not uniform in $[2,3]$ and in $[8,10]$ intervals. Note that occurrences of measurements are larger on the dayside than on the nightside. The dayside-nightside asymmetry results partly from the solar wind flow on the magnetosphere, which squeezes dayside magnetic field lines and stretches nightside magnetic field lines (as illustrated in Fig. 1). The region at $L<10$ is thus more frequently sampled in the dayside. Another factor is the duration of the second analysed period (1 January 2007 to 30 April 2009), which does not cover three full years and happens to favour the dayside MLT sectors. Finally, and this is likely the most important reason, nightside sectors are less populated than dayside ones (Carpenter and Anderson, 1992). Consequently, the WHISPER instrumental limit is more often reached on the nightside of magnetosphere. The meridian projection (Fig. 4b) shows the latitudinal coverage over all MLT sectors. Note the presence of two arms due to the orbit drift effect. The outer arm (larger $L$-values at low latitude) corresponds to the first analysed period, i.e. 2002-2003, and the deeper one covering a larger interval in $L$-values near the equator corresponds to the 2007-2009 time period. In the Northern Hemisphere (MLat $<50^{\circ}$ ) and for large $L$-values ( $L \geq 8$ ), another zone covered by Cluster appears. This zone corresponds to the observations made during the second crossing of the plasmasphere for perigee passes analysed after mid 2008.

\section{Refilling process of the plasmasphere under quiet and steady magnetic activity}

Our main objective in this section is to examine the plasmasphere refilling process by presenting and discussing global density maps, sorted out according to magnetic activity conditions preceding measurements. Two main behaviours are expected during prevailing quiet magnetic activity: the plas- masphere expansion up to large $L$-values (co-rotation dominating in a region covering larger geocentric distances than prior to refilling), and the replenishment of flux tubes from their footprint at ionospheric altitudes to their apex. The two mechanisms are not independent, since a refilled plasmasphere results from replenishment of flux tubes co-rotating in a region dominated previously by convection. The refilling of a magnetic flux tube from sunlit ionosphere has been modelled by assuming steady conditions at sources (Singh and Horwitz, 1992; Wilson et al., 1992). These models point to refilling durations which depend highly on the $L$-value considered, from approximately less than a day at $L<4$, to several days at $L>6$. Such durations are comparable to observed values quoted in Sect. 1 .

Over long-term intervals, the ionospheric source linked to a magnetic flux tube in co-rotation is switched off in the night sector, which increases the overall refilling duration. The consequence of the equatorial drift pattern on the refilling process is clearly illustrated by daily variations of the quiet plasmasphere density observed from the geosynchronous orbit (Reynolds et al., 2003). One can note that the dayside outer plasmasphere region is under replenishment quasi-permanently, whatever the fate of flux tubes when they reach the afternoon sector, co-rotation or sunward convection drift (Décréau et al., 1982). The dayside outer plasmasphere constitutes thus a reservoir of fresh cold plasma, a part of which can later be eventually aggregated to the main plasmasphere body.

\subsection{Classification of density profiles}

In order to elaborate datasets representative of the inner magnetosphere in the course of the refilling process, we sort out density profiles according to magnetic activity conditions prior to measurements. From the above discussion, we decide to compare density variations at time intervals of a couple of days. We use a $3 \mathrm{~h}$ planetary geomagnetic index to characterise the level of activity in the magnetosphere. Applying the same sorting rule to all density profiles, whatever the MLT sector they belong to, allows the dependence of the plasmasphere replenishment to be investigated in local time and in latitude, and at a large time scale. In practice, we characterise the magnetic activity level by the value of the ap index, which has the advantage of being expressed quantitatively, in $\mathrm{nT}$, and is based on data provided by a set of specific stations used to derive the well known Kp index (Mayaud, 1980; Menvielle and Berthelier, 1991). We opt for a simple criterion to define a quiet dynamical state of the magnetosphere: ap values steadily lower than or equal to $15 \mathrm{nT}(\mathrm{Kp}=3)$. This limit has been chosen in reference to $\mathrm{Tu}$ et al. (2006), who studied a case of filled plasmasphere characterised by a Kp index value less than 3 during the preceding 5 days. Although this threshold is considered to be indicative of moderate (rather than quiet) activity, we describe here the magnetosphere as being active (or activated) when 
ap exceeds $15 \mathrm{nT}$, and being quiet otherwise. In practice, an entire density profile (all density records belonging to a given perigee pass) is sorted out as follows: we examine ap time series backward from the start time, starting from ap0 (at the time of the first density sample in the profile), and we note ap1 the first occurrence of ap exceeding the $15 \mathrm{nT}$ limit. Let us call Dq the duration of the time sequence between ap1 and ap0, in unit of hours (Dq is a multiple of 3, and can be equal to 0 ). We sort out the density profiles in three classes, class 1 when $\mathrm{Dq}<48$, class 2 when $48 \leq \mathrm{Dq}<96$, and class 3 otherwise (when $\mathrm{Dq} \geq 96$ ).

In a second step, we examine the geomagnetic conditions attached to each density profile. Actually, selected density profiles are recorded over several hours, typically 8 to $10 \mathrm{~h}$, wherein magnetic activity can change. We keep density profiles for which ap index during the corresponding time interval does not exceed $15 \mathrm{nT}$, discarding thereby 243 density profiles (among them, the ones for which ap0 $>15 \mathrm{nT}$ ). Following this selection, 575 density profiles belong to class 1 , 245 to class 2 and 276 to class 3 . All selected density profiles are acquired during quiet geomagnetic conditions, in the aftermath of an active event. In order to better appreciate what is the delay between the active event and the start of the density profile, we have examined the distribution of Dq values in each class. Average delays are respectively less than a day ( $\sim 16 \mathrm{~h}$ ) for the first class, about three days $(\sim 70 \mathrm{~h})$ for the second class, and more than six days $(\sim 151 \mathrm{~h})$ for the third class. The delays are distributed reasonably smoothly over the available intervals. In order to simplify the wording in the rest of the paper, we will refer to data of the first class as representative of the situation "after one day" (of quiet geomagnetic conditions), to the data of the second class as representative of the situation "after three days" and, finally, to the data of the third class as representative of the situation "after six days".

Finally, let us recall that the classes defined above correspond to a long-term view of plasma evolution in the inner magnetosphere, in contrast with the short-term evolution of the magnetic field considered during the time interval of the density profile, as presented in Sect. 2.3. To our opinion, even if the density (the content) builds up in the long term, it is important to survey the evolution of magnetic flux tubes (the container) in the short term, considering in particular that according to our selection criteria some density profiles are covered during moderate activity conditions such that the geomagnetic field configuration may vary significantly during the acquisition of the profile.

\subsection{Global maps of density distribution}

As indicated in Sect. 2.3, three magnetic field coordinates, $L$, MLT and MLat, have been attached to each density record by use of the magnetic field model tuned to the solar wind conditions related to the time of record. Figure 5 displays quantities representative of the subset of density records measured inside given magnetic flux tubes. The quantities are projected along field lines onto the magnetic equatorial plane, and presented as a succession of equatorial maps, in the $[2,10] \mathrm{L}$ range, at a resolution of 0.5 in $L$ and $0.5 \mathrm{~h}$ in MLT. This procedure combines records taken in slightly different positions in physical space for a same $L$, MLT and MLat position, since the chosen magnetic field model is a dynamical one. However, it responds better to the concept of frozen-in field lines than a procedure using a static model would have.

Each column in Fig. 5 corresponds to one of the three classes defined in Sect. 3.1 above, from left to right: after one day, after three days and after six days of quiet geomagnetic conditions. Each row displays a different parameter, extracted from every $L$-MLT cell. The first parameter (panels a, $\mathrm{b}$ and c) is the number of $n_{\mathrm{e}}$ measurements. The second one is the relative occurrence of densities above $80 \mathrm{~cm}^{-3}$, expressed as a probability $p$ (panels $\mathrm{d}$, e and f). We arbitrarily assume that the density is above $80 \mathrm{~cm}^{-3}$ for all records with the qualitative "high" density information $\left(F_{\mathrm{UH}}>80 \mathrm{kHz}\right)$. The third parameter is the median density value (panels $\mathrm{g}$, $\mathrm{h}$ and i). We thus attribute the same weight to all density records along a given magnetic flux tube, as would be justified for a flat distribution in latitude. The cells coloured in grey in the bottom views represent the portion of the plasmasphere dominated by high-density values (median density value above $80 \mathrm{~cm}^{-3}$ limit). This region matches exactly cells in the second row where the colour (from greenish to red) indicates a probability $p$ greater than 0.5 . The median parameter is more appropriate than the usual mean parameter to represent the density in a $L$-MLT cell since cells contain both quantitative information (density values) and qualitative information (namely that the density is above the $80 \mathrm{~cm}^{-3}$ limit).

When interpreting the results presented in Fig. 5, one must keep in mind the major weaknesses of the exercise, i.e. the fact that the magnetic flux tubes are not evenly nor fully sampled by the available orbit passes, on one hand, and that, on the other hand, data gaps are present on some orbits, as described in Sect. 2. Nevertheless, it still enables presenting an approximated view of the plasmasphere projected onto the equatorial plane, and, above all, comparing views obtained under similar sampling conditions, but at different steps of the refilling process. In spite of the uneven coverage of $L$-shells (top views), the maps of Fig. 5 point out indeed some interesting features. The first feature (second row) is the presence of abrupt changes when following increasing $L$-values at a given MLT in the value of the probability $p\left(n_{\mathrm{e}}>80 \mathrm{~cm}^{-3}\right)$. It indicates that the sharp density gradients observed in individual density profiles (see example in Fig. 2) are all grouped at a similar $L$-value for a given MLT sector and a given category (at least for most of them). Those $L$-values can be considered as positions of an "instantaneous" PBL assumed to migrate, with increasing time, between initial PBL positions (closer to Earth) and final PBL positions (farther from Earth). It is convenient to mark the change in probability values at $p=50 \%$, which corresponds 
$\mathrm{Dq}<48$
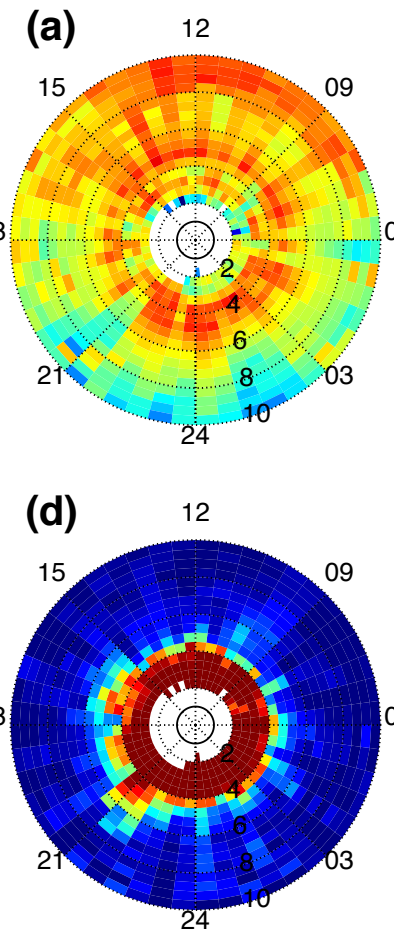

(g)

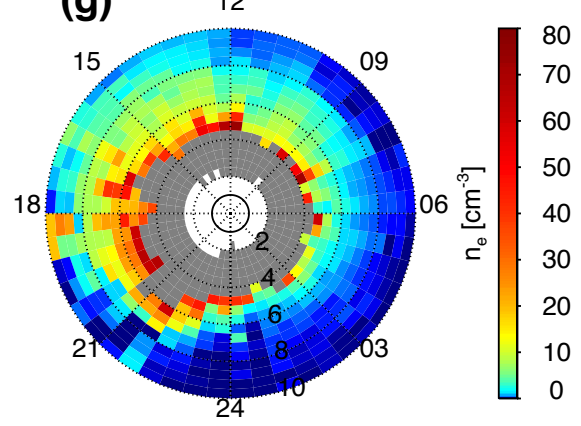

$48 \leq \mathrm{Dq}<96$

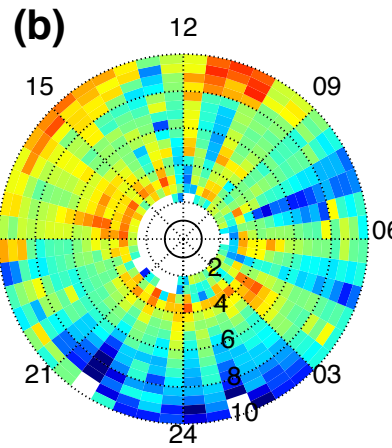

(e)
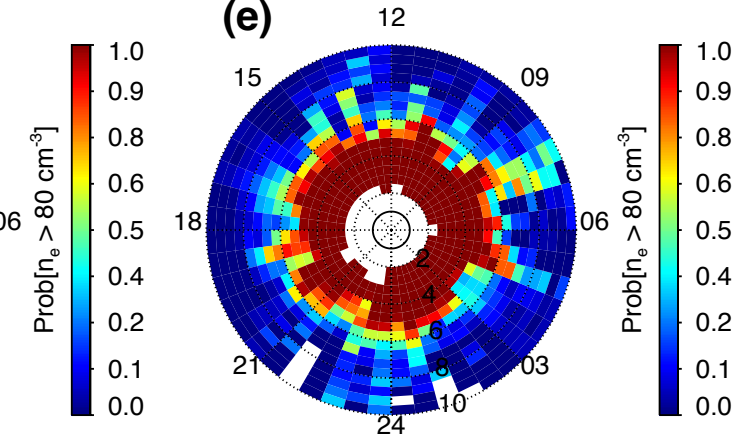

(h)

12

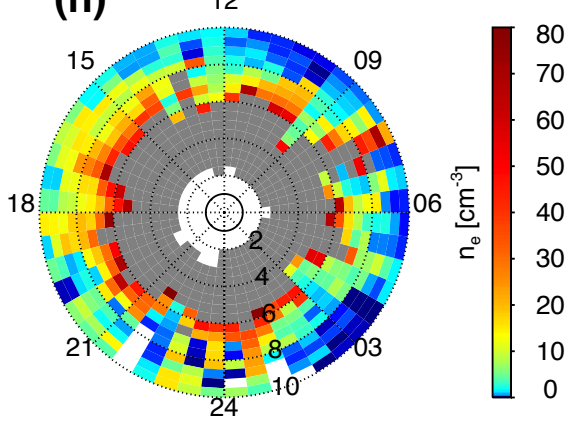

$\mathrm{Dq} \geq 96$

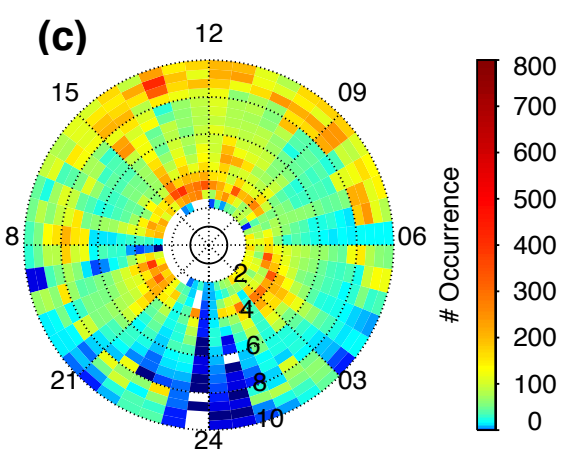

Fig. 5. Electron density information inside magnetic flux tubes intersecting equatorial plane at a given $L$ and MLT are shown in $L-M L T$ views, using a resolution of $0.5 \mathrm{in} L$ and $0.5 \mathrm{~h}$ in MLT. The first row shows the distribution of the occurrence of $n_{\mathrm{e}}$ measurements in each class. The second row displays the relative number of measured density values above $80 \mathrm{~cm}^{-3}$, expressed in terms of probability. The third row displays the median value of $n_{\mathrm{e}}$. The electron density median is colour-coded on a linear scale and grey colour corresponds to the portion of the plasmasphere dominated by high density values $\left(>80 \mathrm{~cm}^{-3}\right)$. The three columns display measurements obtained under the three conditions defined in the study, namely $0 \leq \mathrm{Dq}<48$ (panels a, $\mathbf{d}$ and $\mathbf{g}$ ), $48 \leq \mathrm{Dq}<96$ (panels $\mathbf{b}, \mathbf{e}$ and $\mathbf{h}$ ) and Dq $\geq 96$ (panels $\mathbf{c}, \mathbf{f}$ and i) of continuous quiet activity, where Dq is expressed in unit of hours.

to the outer limit of regions painted in grey in the bottom views of Fig. 5. In each category, the shape of the grey region is representative of the shape of the plasmasphere in formation. It is roughly circular for the three data classes, with a radius increasing from $L \sim 4$ in the first class (after one day of quiet conditions) to $L \sim 6$ in the second class (after three days) and $L \sim[6.5,7]$ in the third class (after six days). It appears thus that the main increase in the $L$ size of the plasmasphere occurs during the first three days of quiet geomagnetic conditions, and that this size increases further after that, but slowly and slightly.

The bottom views (panels g to i in Fig. 5) emphasise the $L$ MLT distribution of density values measured in the intermediate range by WHISPER (i.e. $0.2-80 \mathrm{~cm}^{-3}$ ). Green to yellow colours, easily recognisable, cover the restricted range of density values $\left(5-15 \mathrm{~cm}^{-3}\right)$ typical of the dayside plasmasphere reservoir mentioned above (Décréau et al., 1982). For the first class $(\mathrm{Dq}<48)$, densities in the WHISPER range are 
present in all sectors, up to high $L$-values $(L \sim 8)$, except in the midnight sector where they are seen up to $L \sim 5$. The general shape drawn by median densities at $n_{\mathrm{e}}=10 \mathrm{~cm}^{-3}$ (green to yellow colour code) is similar to the shape of the statistical outer boundary of measurable ions presented in Horwitz et al. (1990) (their Fig. 5a). A bulge of high densities $\left(n_{\mathrm{e}} \sim 50 \mathrm{~cm}^{-3}\right)$ shows up in the dusk sector $(15: 00-$ 21:00 MLT range). This is the region where, according to a classical interpretation, the co-rotation electric field and the convection electric field are equal and opposite (Carpenter, 1967; Chappell et al., 1970; Higel and Lei, 1984). Note also the local increase of the plasmapause-like boundary at 21:00 MLT (patch in grey colour up to $L=6$ ). This MLT sector is where the bulge region is installed under very low activity (e.g. Carpenter, 1970; Maynard and Grebowsky, 1977).

After 3 days of low activity (Fig. 5h), density values in the WHISPER range are observed in a large part of the $L$-MLT domain, with a smaller zone of very low density (dark blue) in the night sector than after one day. High-density values are present, irregularly, up to high $L$-values ( $L \sim 8$ and above) in all MLT sectors. After six days of low activity (Fig. 5i), the presence of high densities up to $L=8$ and above is observed more systematically than for class 2 , particularly in the 10:00-22:00 MLT sector.

We examine now $L$-MLat maps of the median electron density for the three classes of geomagnetic conditions defined above, and we present the results in Fig. 6, where each column corresponds to a given class. Four maps are elaborated with data from each class, grouping density records acquired in four MLT sectors, respectively, around midnight $(21: 00 \leq \mathrm{MLT}<03: 00$, first row $)$, around dawn $(03: 00 \leq$ MLT $<09: 00$, second row $)$, around noon (09:00 $\leq$ MLT $<15: 00$, third row) and around dusk (15:00 $\leq$ MLT $<21: 00$, fourth row). Figure 6 is constructed in a similar way to Fig. 5: all density records from a dataset belonging to the selected class (of magnetic activity) and belonging to a given cell in $L$ and MLat value (of dimension 0.2 in $L$ and $2.8^{\circ}$ in MLat, respectively) are grouped in a data packet, whatever their MLT value in the global sector considered. Positive and negative latitudes are grouped in the same cell, assuming symmetry in term of density between both hemispheres. Indeed, density structures derived from a first $L$-MLat mapping (not shown) appear as globally similar in each hemisphere. Joining data from both hemispheres allows a smooth coverage of a large $L$-MLat zone covered partially in each hemisphere (see Fig.4b, showing the $L$-MLat orbital coverage). The question of inter-hemispheric symmetry will be investigated in the future. The median value of density in the data packet is shown using the same colour code as in Fig. 5, third row. Note that these views, like Fig. 4b, are presented in a different coordinate system than the familiar meridian views where $L$-shells are drawing closed contours passing through the centre of the Earth (see for instance Laakso et al., 2002; Huang et al., 2004; Pierrard and Stegen, 2008). Here, $L$-shells are drawing geocentric circles
( $L$-values indicated on horizontal axes). Constant geocentric distance (dashed-dot curves), in the frame of a dipolar field assumption, are superimposed ( $R$-values in Earth radii equal to $L$-values at equator).

One can distinguish three different zones in the maps of Fig. 6. The first one, coloured in grey, is characterised by densities above $80 \mathrm{~cm}^{-3}$. The second zone is characterised by densities in the WHISPER range (colour scale from blue to red). The third one is of a different nature. It is the blank zone, of variable extension in latitude, seen near the equator around $L \sim 6$. The latter zone is hidden in the maps of Fig. 5. It is due to a lack of orbital coverage, on the one hand (see Fig. 4b), and, on the other hand, to the presence of plasma of low density ignored in our dataset (explaining the larger blank zone in night side MLT sectors). Whatever their nature, the blank zones, covering regions at the apex of magnetic flux tubes where density values are likely lower than nearer to Earth, bias the statistical results shown in Fig. 5; some median densities plotted in this figure are overestimations of actual median densities.

The shape of the two first zones in the $L$-MLat maps presents characteristics forming novel information. The extension of the first zone (high densities marked in grey colour) varies clearly with respect to the duration $\mathrm{Dq}$ of quiet magnetic activity, whereas there is no significant variation with the MLT sector considered. This is in agreement with the almost circular shape of the grey zone in the maps of panels (g) to (i) in Fig. 5. For the first class (after one day of low activity), maps of panels (a), (d), (g) and (j) in Fig. 6 display a grey zone occupying geocentric distances below $R \sim 3 R_{\mathrm{E}}$ at $L \geq 5$ (note that $R$ is deduced from the conversion of the $L$-MLat position in geocentric distances, according to a simple dipolar model). Below $L \sim 5$, the grey zone extends at higher geocentric distances, up to $R \sim 4 R_{\mathrm{E}}$ and even up to $R \sim 5 R_{\mathrm{E}}$, in a patchy manner in the dusk sector and near the equator. The higher altitude of plasmas with $n_{\mathrm{e}}>80 \mathrm{~cm}^{-3}$ inside an $L$-shell of $L \sim 5$ (than outside) is an indication of a plasmasphere in formation. In this region $(L<5)$, a density decrease with decreasing magnetic latitude is visible for $L>4$, as happens on partially empty magnetic flux tubes (Wilson et al., 1992), whereas at $L<4$ the information available in our dataset (median density larger than $80 \mathrm{~cm}^{-3}$ ) does not allow distinguishing partially empty from fully replenished flux tubes. For the second class of our dataset (after three days of low activity, panels b, e, h and k in Fig. 6), the grey zone occupies only geocentric distances below $R \sim 3 R_{\mathrm{E}}$ at $L \geq 6$. Inside an $L$-shell of $L \sim 6$, the plasmas with $n_{\mathrm{e}}>80 \mathrm{~cm}^{-3}$ extend at higher altitudes (up to $R \sim 5 R_{\mathrm{E}}$ ), indicating a plasmaphere in formation inside a larger volume than after one day. There is some indication of partially empty magnetic flux tubes near the equator in the dawn and noon sectors (panels e and $\mathrm{h}$ in Fig. 6), i.e. a density decrease when following the flux tube at $L \sim 5.5$ from high latitudes toward the equator. However, the blank zone near the equator above $L=5$ prevents getting a full picture 


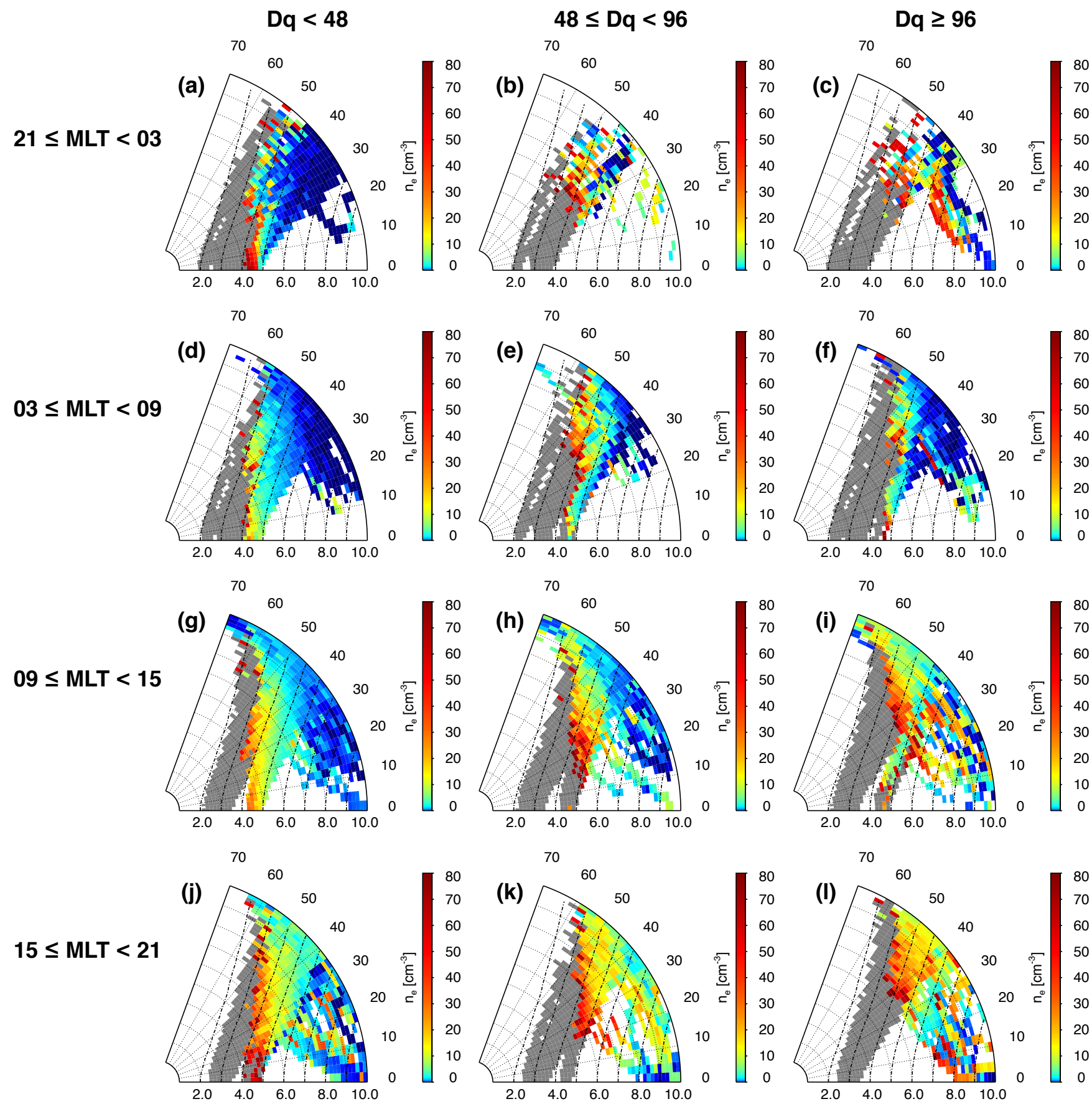

Fig. 6. $L$-MLat views of the median value of densities measured at a given $L$-shell and magnetic latitude (defined in absolute value) using a resolution of 0.2 in $L$ and $2.8^{\circ}$ in MLat. Datasets combine density records over large local time sectors, 21:00-03:00 MLT (first row), 03:00-09:00 MLT (second row), 09:00-15:00 MLT (third row) and 15:00-21:00 MLT (fourth row). The three columns are defined as in Fig. 5. Colour codes are defined as in Fig. 5, second row. Iso- $R$ curves, where $R$ is the geocentric distance derived from the $L$-MLat position, according to the dipolar model, are also superimposed (dashed-dot lines) in order to guide the reader.

of this behaviour. Finally, the third class of our dataset (after six days, panels c, f, i and j in Fig. 6) displays similar characteristics: a plasmasphere in formation inside a still higher $L$-shell $(L \geq 6.5)$.
Maps of Fig. 6 show that median densities in the WHISPER range (the second zone) are placed outward of the grey zone (median $n_{\mathrm{e}}>80 \mathrm{~cm}^{-3}$ ), as described above. This region is characterised in better detail than the first zone, with a colour code pointing to various density levels. The 


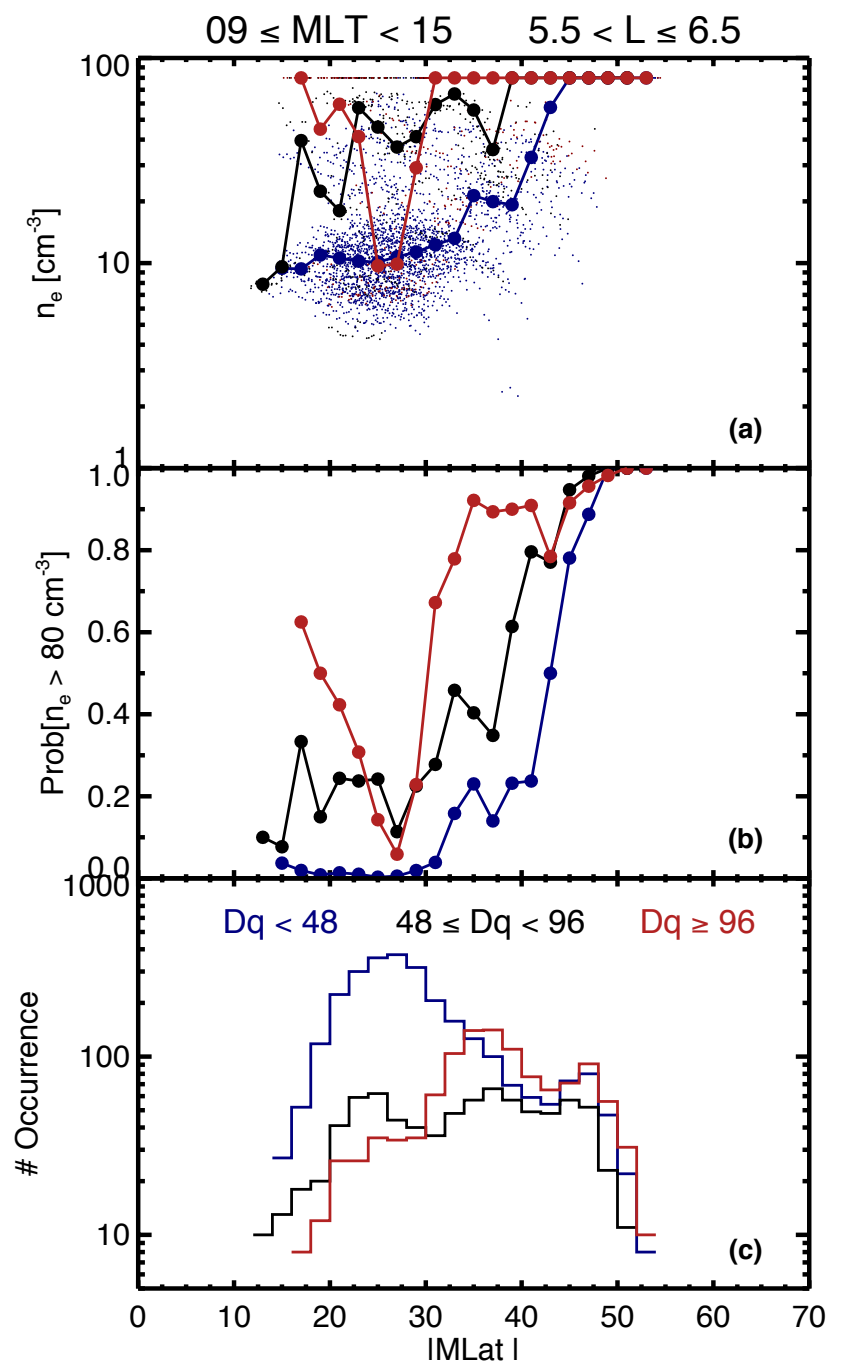

Fig. 7. Distribution of electron density versus latitude (at a resolution of $2^{\circ}$ ), for magnetic flux tubes covering the 09:00-15:00 MLT sector and crossing magnetic equator at $5.5 \leq L<6.5$. Panel (a) displays the scatter plot of $n_{\mathrm{e}}$ records (dots) and the corresponding median values (full circles). Panel (b) displays the probability of observing densities greater than $80 \mathrm{~cm}^{-3}$ and panel (c) shows occurrence numbers. For all panels, the three curves depict properties of $n_{\mathrm{e}}$ for a duration of quiet activity being $\mathrm{Dq}<48$ (blue), $48 \leq \mathrm{Dq}<96$ (black) and $\mathrm{Dq} \geq 96$ (red), as defined in the text.

maps display a clear evolution of this zone, not only with the duration Dq (i.e. a displacement with Dq, which is a consequence of the spatial extension of the first zone with Dq) but also an evolution with the MLT sector considered. The MLT dependence for the first class of magnetic activity (maps in the left column) is two fold: (i) the boundary layer, i.e. the zone coloured from red to green, narrows in the midnight sector (Fig. 6a), and gets thicker and thicker with increasing central MLT (panels $d, g$ and $j$ in Fig. 6); (ii) a zone with patchy cells at high densities (red or yellow colour) is present above a geocentric distance $R \sim 6 R_{\mathrm{E}}$ in the noon and dusk sectors (panels g and j, Fig. 6), whereas the midnight and dawn sectors (panels a and d, Fig. 6) indicate densities either low (blue colour) or not measurable (blank zone) in this area. For the second and third classes of activity (maps shown, respectively, in the central and right columns of Fig. 6), one can note the presence of small regions of high or medium densities in the night and dawn sectors at high altitudes and high $L$-values $\left(R>6 R_{\mathrm{E}}, L>7\right)$, which are not seen in the left column. The patchy cells of high or medium densities cover a larger area in the noon and dusk sectors than in the night and dawn sectors (panels $\mathrm{h}, \mathrm{i}, \mathrm{k}$ and $\mathrm{l}$ compared to panels b, c, e and $\mathrm{f}$ in Fig. 6). Moreover, the noon and dusk sectors display a large area of intermediate densities (yellow to red colours) at high magnetic latitudes and $L$-values. In summary, both the "plasmasphere" $\left(n_{\mathrm{e}}>80 \mathrm{~cm}^{-3}\right)$ and the neighbouring "trough" ( $n_{\mathrm{e}}$ in the WHISPER range) expand in volume with the duration of low activity, and mainly in the noon and dusk sectors. Our dataset does not cover all key areas of the $L$-MLat map but it seems that, even after six days, the apex of flux tubes is still partially empty above $L \sim 6$.

\subsection{Detailed density distribution along magnetic flux tubes and near equatorial plane}

We now present further details of the density distribution, shown in Figs. 7 to 9, where individual measured density values are visible. This allows the dispersion of the measured densities to be appreciated around the median values calculated inside chosen cells of the $L$-MLat - MLT domain. Figure $7 \mathrm{a}$ presents the distribution of density values (thin dots) covering the sector 09:00-15:00 in MLT and the range 5.5 to 6.5 in $L$-values, as a function of the magnetic latitude MLat, for the three classes of magnetic activity history. The resolution in MLat is $2^{\circ}$. A given colour code attributed to each class allows a direct comparison of the median density values (thick dots), with same values presented (at a better resolution in $L$ ) in the $L$-MLat panels (g) to (i) in Fig. 6. Figure 7 displays also the percentage of observation above $80 \mathrm{~cm}^{-3}$ limit (panel b) and the total number of density records in each data subset (panel c).

Above about $45^{\circ}$ MLat, all three classes show the same characteristics: a majority of density values above $80 \mathrm{~cm}^{-3}$, and a similar total number of records. Between $35^{\circ}$ and $45^{\circ}$ latitude, the number of records is significant in all classes and the percentage of high values increases with increasing refilling duration. This behaviour is consistent with a view where the total content of a flux tube increases with the duration of refilling. It indicates that, around $L=6$, the refilling process is still active after three quiet days. Below $30^{\circ}$ MLat, the probability $p$ for class 3 , as well as the density profile, display an irregular behaviour, which is likely due to the small number of records.

In spite of the lack of coverage near the equator in the datasets available for this study, the three profiles of density versus magnetic latitude shown in Fig. 7a present 
quantitative features which can be compared to published models and observations. For the first class of data, the scatter plot is reasonably compact around median density values. The latitudinal profile of median densities draws a concave shape, qualitatively similar to what is obtained in a collisional kinetic models, after the first hours of refilling of initially empty flux tubes, and during the subsequent time interval, when ions diffuse via collisions (Wilson et al., 1992). Near the equator, the profile $n_{\mathrm{e}}$ (MLat) is almost flat, then the slope increases with MLat, presenting a step-like shape in the range $\left[35^{\circ}, 45^{\circ}\right]$. The median value obtained at the lowest latitude available $\left(15^{\circ}\right)$ is $n_{\mathrm{e}} \sim 8 \mathrm{~cm}^{-3}$, equal to the density calculated at $L=6$ with the above quoted model. This value is also comparable to values observed on board ISEE in the dayside trough (Carpenter and Anderson, 1992), i.e. $n_{\mathrm{e}}$ in the $[4,9] \mathrm{cm}^{-3}$ interval. For the second class of data (after three days), the number of density records near the equator (MLat $<20^{\circ}$ ) is too scarce for median values to be discussed quantitatively. In the $\left[20^{\circ}, 25^{\circ}\right]$ MLat interval, median values (in the $[20,60] \mathrm{cm}^{-3}$ interval) are comparable to the value of $30 \mathrm{~cm}^{-3}$ obtained in the model of Wilson et al. (1992) at the equator after a refilling duration of $48 \mathrm{~h}$. Finally, the median value obtained at MLat $=30^{\circ}$ for the third class (after six days) is greater than $80 \mathrm{~cm}^{-3}$, which complies with the equatorial densities $n_{\mathrm{e}} \sim 100 \mathrm{~cm}^{-3}$ observed at $L=6$ in the dayside saturated plasmasphere (Carpenter and Anderson, 1992). More generally, the density values belonging to class 2 and class 3 are similar to values obtained by in situ or whistler observations after an extended quiet period (Corcuff et al., 1972), while the density values belonging to class 1 correspond to density values characteristic of the permanent dayside reservoir (Décréau et al., 1982).

Figure 8 displays the same quantities as shown in Figs. 7a and 7c, but for higher $L$-values, i.e. $7.5<L<8.5$, and in four MLT intervals covering all MLT sectors. Scatter plots and median values are displayed on the left side of the figure, and matching occurrences on the right side. Successive MLT sectors are displayed from top to bottom, with increasing hours: 21:00-03:00 MLT, 03:00-09:00 MLT, 09:00-15:00 MLT and 15:00-21:00 MLT. At high latitudes (MLat $>50^{\circ}$ ), the density profiles are only indicative. Indeed, the gyrofrequency is important and the qualitative density information (densities placed at $n_{\mathrm{e}}=80 \mathrm{~cm}^{-3}$ in the scatter plot) is not reliable (see Sect. 2.1).

The density variations versus MLat shown in Fig. $8 \mathrm{c}$ can be compared to the density profiles shown in Fig. 7, top panel. This allows the result of the refilling process to be appreciated in the same MLT sector, but along magnetic flux tubes at two different $L$-values. For the first class of magnetic activity (blue curves), the two profiles are qualitatively similar: almost flat near the equator, they display a larger slope in density at high latitudes, above, respectively, at MLat $\sim 40^{\circ}$ for $L \sim 6$ (Fig. 7) and at MLat $\sim 50^{\circ}$ for $L \sim 8$ (Fig. 8). These magnetic latitudes, when converted in geocentric distances - using the Tsyganenko model shown in Fig. 1 - lead to similar geocentric distances of, respectively, $3.6 R_{\mathrm{E}}$ $(L=6)$ and 4.0 $R_{\mathrm{E}}(L=8)$. Quantitatively, the median density near the equator is $n_{\mathrm{e}} \sim 2 \mathrm{~cm}^{-3}$ at $L \sim 8$ (compared to $n_{\mathrm{e}} \sim 8 \mathrm{~cm}^{-3}$ at $L \sim 6$ ), in agreement with the "trough" equatorial density value published by Carpenter and Anderson (1992). Median densities increase with the duration Dq of low activity, but in a less spectacular way than at $L \sim 6$. Among the very dispersed values seen in the scatter plot of data belonging to class 3 (red dots, Fig. 8c), the highest ones, $n_{\mathrm{e}} \sim 40 \mathrm{~cm}^{-3}$ (at MLat $\sim 20^{\circ}$ ), are comparable to the highest densities encountered by ISEE 1 in the dayside "saturated" plasmasphere near the equator, at $L=8$ (Carpenter and Anderson, 1992).

More generally, the density distribution displayed in all four MLT sectors of Fig. 8 can be described as follows:

1. Two main types of $n_{\mathrm{e}}$ (MLat) profiles are showing up. The first type, which we will refer to as a "trough" like profile (for example, all median densities in class 1), displays a concave shape, shallow near the equator. The second type, which we will refer to as a "saturated plasmasphere" like profile, displays a flat shape. It is formed by the highest density values in MLat bins of the scatter plots of class 3. These two types reproduce modelled behaviours, respectively, in partially empty flux tubes and in saturated flux tubes (Wilson et al., 1992);

2. Trough like profiles display density values increasing with MLT sectors presented from top to bottom panels. At MLat $=30^{\circ}$, densities from smoothed profiles are, respectively, $0.9 \mathrm{~cm}^{-3}$ in the midnight sector, $1.5 \mathrm{~cm}^{-3}$ in the dawn sector, $2.5 \mathrm{~cm}^{-3}$ in the noon sector, and $5 \mathrm{~cm}^{-3}$ in the dusk sector. In contrast, densities in "saturated profiles" cannot be valuably compared from one MLT sector to the next. All of them display similar values $\left(n_{\mathrm{e}} \sim 60 \mathrm{~cm}^{-3}\right)$, more than an order of magnitude above trough values;

3. When the occurrence of measurement is sufficient to compare median densities of the three different classes, the increase of density with the duration Dq is modest. An increase of the order of a factor two after several days can be noted at MLat $=45^{\circ}($ panels b, c, d);

4. The huge dispersion of density values in a same MLT $L$ - MLat cell, for the three classes of magnetic activity, indicates that the chosen criteria for sorting out the dataset is not very efficient. More selective criteria, for instance choosing a smaller ap value to define low activity, could be worked out. As an example, the high densities measured in the night sector (Fig. 8a) correspond to an event (15 November 2008) with ap $\leq 4$ during five consecutive days;

5. Irregular density profiles are observed near the equator $\left(\right.$ MLat $\left.<25^{\circ}\right)$. 

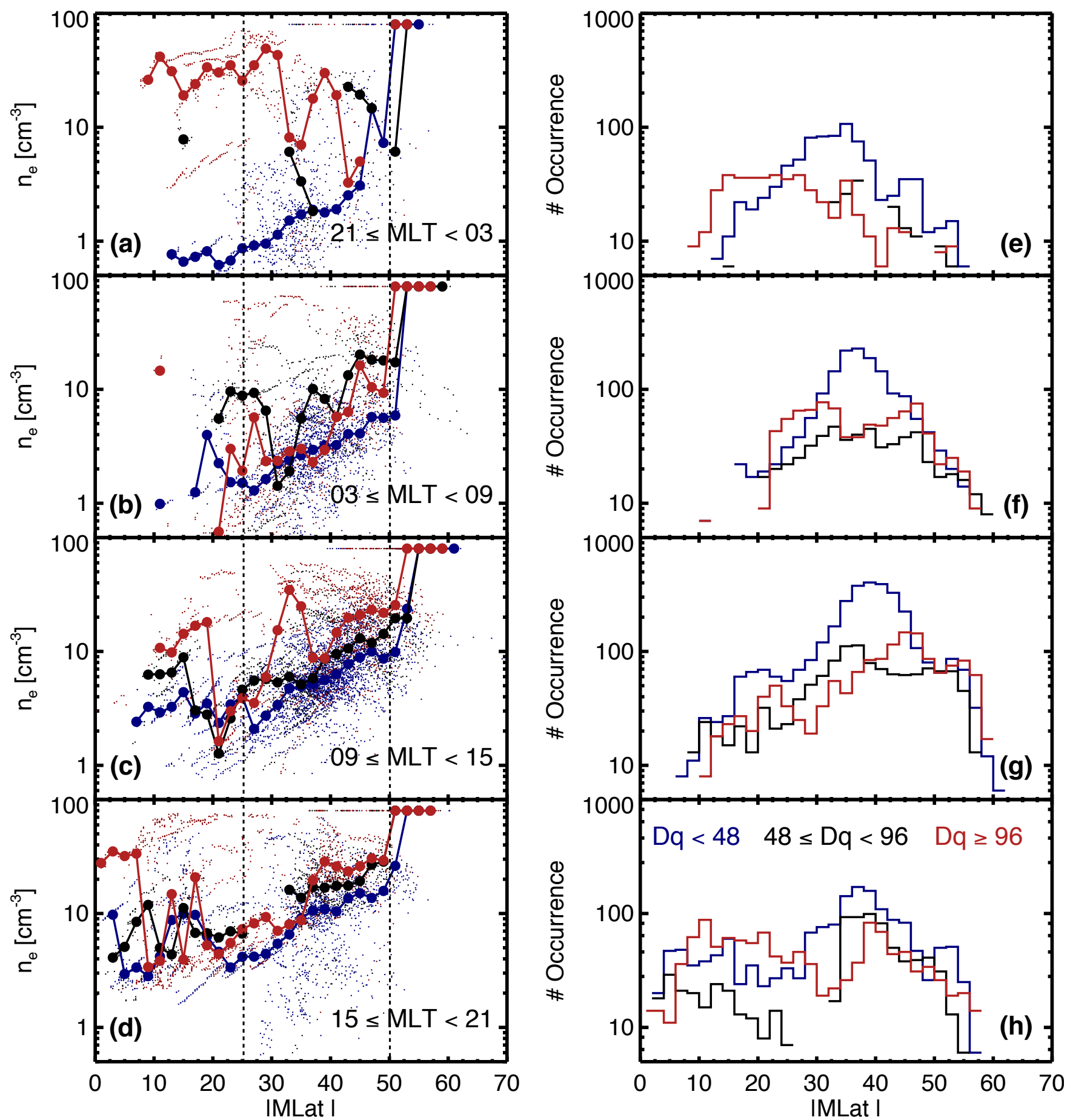

Fig. 8. Same as Fig. 7a (left column) and Fig. 7c (right column), but for field lines at higher $L$-values, $7.5 \leq L<8.5$. Scatter plot (left) and occurrence numbers (right) are split in four MLT sectors, as in Fig. 6: 21:00-03:00 MLT (panels a and e), 03:00-09:00 MLT (panels b and f), 09:00-15:00 MLT (panels $\mathbf{c}$ and $\mathbf{g}$ ) and 15:00-21:00 MLT (panels $\mathbf{d}$ and h).

The objective of Fig. 9 is to visualise equatorial density profiles, $n_{\mathrm{e}}(L)$, after different refilling durations, and over four MLT sectors, using the same presentation as Fig. 8. Left panels display scatter plots and median densities at a resolution of 0.25 in $L$, and right panels display occurrences.
The domain covered by the density records presented in each panel is a slice in magnetic latitude close to equator $\left(-30^{\circ}<\right.$ MLat $\left.<30^{\circ}\right)$, and a range of $6 \mathrm{~h}$ in MLT. In order to guide the eye, two $n_{\mathrm{e}}$ profiles in $L^{-4}$ are plotted in left panels. This variation of $n_{\mathrm{e}}$ assumes that flux tubes at various $L$ 

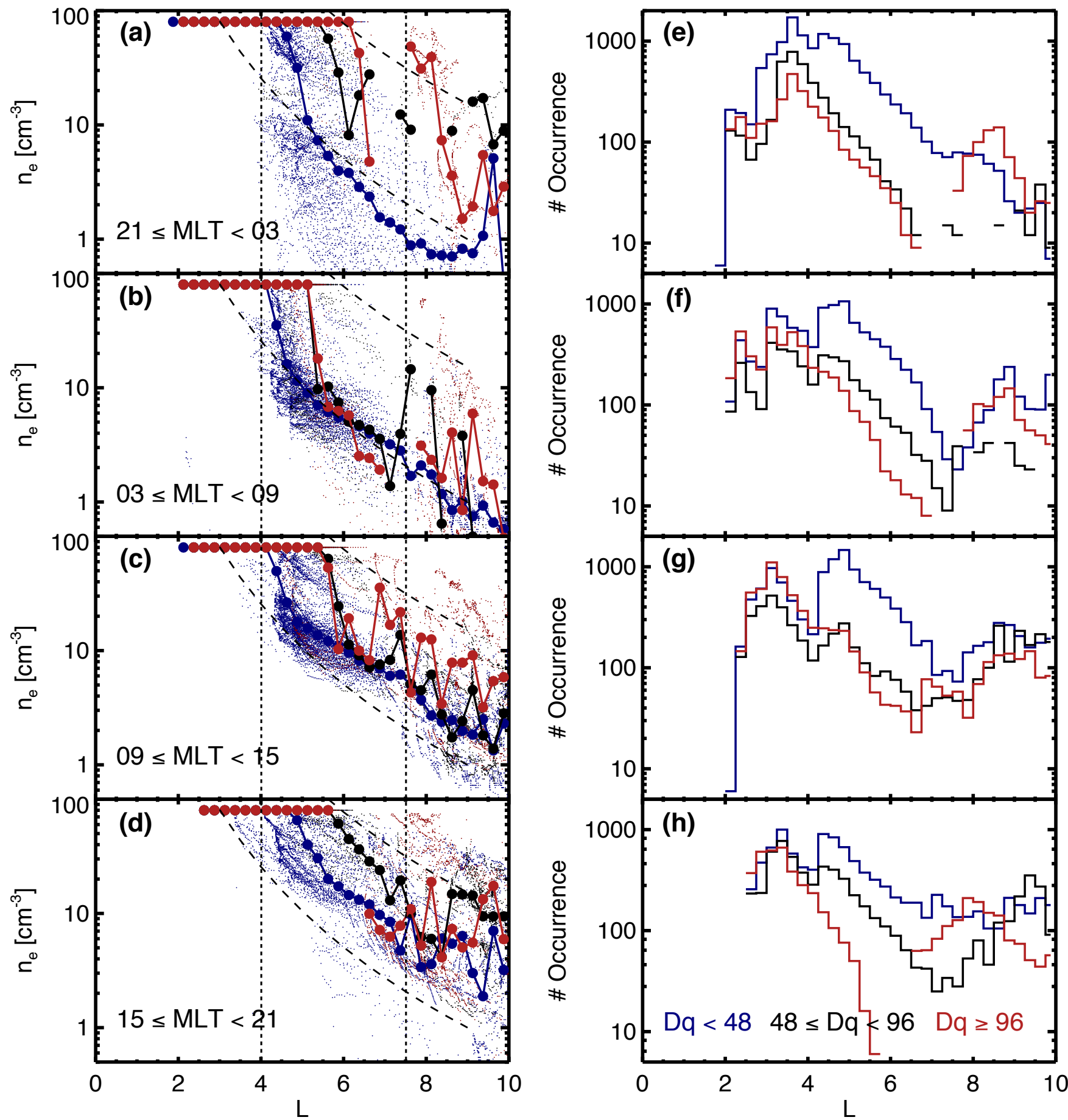

Fig. 9. Distribution of electron density versus $L$, using a resolution of 0.25 in $L$-value, over a region covering the latitudinal range $-30^{\circ} \leq$ MLat $\leq 30^{\circ}$. Panels display identical quantities as in Fig. 8, using the same colour code. Dashed lines show distributions in $L^{-4}$.

carry the same plasma content (noting that their volume vary as $L^{-4}$ ).

The plots shown in Fig. 9 confirm the observed evolution of density with $L$-value described above: (i) a clear increase of density with the duration Dq of low activity for $L$-shells in the lower range $(4<L<6)$ and a smaller increase in the higher range $(6<L<10)$, noting the irregular coverage of the high L-range from occurrences plotted in panels (e), (f), (g) and (h); (ii) a regular increase of density in the trough with central MLT time considered (see evolution from top to bottom, panels a, b, c and d, of median values of class 1 density records). We can add a few additional remarks. The first 
remarks concern the signature of a plasmapause like boundary, i.e. a density step in the profile $n_{\mathrm{e}}(L)$. This step disappears in the dusk sector. This could indicate that, under conditions of low activity, equatorial drift paths are closed up to high $L$-values. In the three other MLT sectors, this step shows up clearly. Its position in $L$ increases with the duration $\mathrm{Dq}$ of low activity. There is no clear dependence of this boundary according to the MLT sector, a feature already mentioned above, in the descriptions of Figs. 5 and 6. Further remarks concern density values in the noon and dusk sector. They are placed inside a slice bounded by two profiles in $L^{-4}$. The lowest values are probably controlled by the dayside reservoir (Décréau et al., 1982), and the highest values by the total volume available inside magnetic flux tubes. In contrast, very low densities are observed in the nightside of the magnetosphere (Fig. 9a).

\subsection{Discussion}

The paradigm we wish to examine here is how, under conditions of a globally stationary equatorial convection pattern (ap $\leq 15 \mathrm{nT}$ after episodes with ap $>15 \mathrm{nT}$ ), the volume of a plasmasphere in formation is getting replenished. This volume is assumed, in a simple picture, to be rigidly bounded (by the last closed equatorial drift path and its projection along magnetic field lines forming a "final" PBL) and at least partially emptied from erosion mechanisms. Supplies (and losses) of matter, at ionospheric level, are expected to bring (or remove) plasma material inside this volume, supplies being on average more effective than losses. Having no preconception about the position of the final PBL, we examine the density distribution over the global volume inside a large $L$-range $[2,10]$. The general questions we wish to address, and try to answer from our density observations, are the following: What is the final shape of the plasmasphere in formation? How does the ionized gas occupy the threedimensional space available? Our ambition, at the same time, is to try and improve our understanding of the physics of the plasmasphere refilling process. We shall now examine our results according to three different aspects of the question addressed above, in turn.

\subsubsection{Final boundary of the plasmasphere in formation}

The $L$-MLT maps in Fig. 5 display the shape of an "instantaneous" PBL (the outward limit of the grey zone), which expands with the duration Dq (see Sect. 3.2). This quasicircular PBL expands of about $2 R_{\mathrm{E}}$ in two days, and of about $0.5 R_{\mathrm{E}}$ during the next three days, which could lead to the interpretation that a "final" boundary, approached asymptotically, is placed next to the largest observed PBL, i.e. at a radius of about $7 R_{\mathrm{E}}$. However, observations presented later in the paper demonstrate that this view is oversimplified. They demonstrate (i) that the volume inside the $L=7$ magnetic shell is far from being filled up to saturation, at least on av- erage, after the longest Dq value considered (Figs. 6 and 9); and (ii) that some events display saturated latitudinal density profiles at $L \sim 8$ (Fig. 8) after five very quiet days. We consider, thus, that we must revise the paradigm stated above. It is likely valid for a selection of events present in our dataset but there is not a large enough number of these to allow the 3-D analysis aimed at. For most of our selected events, the equatorial convection pattern is not stationary, and the "final" boundary is a moving one. As a consequence, our statistical analysis of the 3-D refilling pattern is to be taken as representative of a refilling plasmasphere where plasma losses are not only occurring at ionospheric levels but also at higher altitudes from plasmasphere erosion during episodes of moderate activity.

The effects of a centrifugal force due to co-rotation of the plasma with the angular velocity of the Earth had been brought to attention by Lemaire (1974) as an important mechanism leading to plasmasphere erosion in the dawn sector at $L<5.8$ (Pierrard and Cabrera, 2005). Erosion is due to convective instability inducing interchange motion of flux tubes transverse to equatorial drift paths. Recently, André and Lemaire (2006) have shown that effects of the magnetic curvature on convective instability, when taken into account, are largely dominant over the effects of the effective gravity in the equatorial regions of the plasmasphere. They show that, in addition to transverse interchange motion, translational (along field lines) interchange motion is to be expected. They have shown in particular that the density model derived empirically for a saturated plasmasphere by Carpenter and Anderson (1992), is unstable above $L=6.6$. More generally, André and Lemaire (2006) demonstrate that the plasmaspheric wind is a leakage mechanism active deep inside the plasmasphere. In brief, this recent study provides important hints about physical mechanisms controlling the refilling process of the plasmasphere under realistic conditions, stressing the importance of a 3-D picture.

\subsubsection{Latitudinal distributions}

Quantitative comparisons of latitudinal density profiles (presented in Figs. 6, 7 and 8) with previous studies are difficult since our observations are novel ones, taken under specific conditions and in regions not well explored. We can however examine, at least qualitatively, a few characteristics showing up in the maps and curves presented above, and see if they are present in other studies. All observations presented above (Sects. 3.2 and 3.3) show that the magnetic flux tubes are filling up starting from the ionospheric base, then gradually toward higher altitudes. The same behaviour is reproduced by the theoretical study of Wilson et al. (1992). The shape of the latitudinal density profile that they obtained during the replenishment is comparable, qualitatively, to the latitudinal profiles observed in the trough (Figs. 7 and 8 ) at medium and low latitudes. The profiles gets flat near the equator as soon as the plasmasphere gets fully replenished. The latitudinal 
density profiles observed by Cluster can also be compared to observations by Polar presented in Denton et al. (2002b). In both cases, the shape is more flat near the equator for smaller $L$-values (from $L=7$ to $L=5.5$ in Polar observations; from $L=8$ to $L=6$ in our study). The scatter plots in Fig. 8, when considering only the highest densities in each MLat cell, indicate that flat latitudinal profiles can also be seen at a high $L$-values $(L \sim 8)$, hence that the plasmasphere, under favourable conditions, can get saturated up to this high $L$-range.

We found no direct evidence in other studies of the presence of the density knee in latitudinal profiles along magnetic flux tubes revealed by our observations. Such a knee, or density gradient, at medium to high latitudes is clearly seen in most maps of Fig. 6 near the outward edge of the zone coloured in grey (forgetting regions at radial distances below $3 R_{\mathrm{E}}$ and latitudes above $50^{\circ}$, where high gyrofrequencies affect qualitative results, see discussion in Sect. 2.1). The same feature, revealed in better detail in Figs. 7 and 8, provides the more precise figure of a geocentric distance $R \sim 3.6 R_{\mathrm{E}}$ at $L=6$ and $R \sim 4.0 R_{\mathrm{E}}$ at $L=8$ in the noon MLT sector. In a lower $L$ range $(L<3)$, observations of the sounder instrument on board IMAGE imply the presence of density knees at medium $\left(\sim 40^{\circ}\right)$ latitudes during depletion and refilling processes (Reinisch et al., 2004). A possible common explanation could be based on the convective instability mechanism described in André and Lemaire (2006). Actually, conditions of convective instability are expected to differ according to geocentric distance (meeting different conditions in magnetic field curvature and effective gravity). Referring to the model of Pierrard and Stegen (2008), particles on trapped orbits with mirror points in the same hemisphere (type E in Fig. 1 of the paper) and occupying the lowest altitudes could be less affected by instability, and get populated more rapidly, during the refilling process than trapped orbits with mirror points in two different hemispheres. This could perhaps be checked by allowing the fractions of trapped particles to be a fit function of magnetic latitude, a possibility of improvement of their model suggested by the authors.

\subsubsection{Density distribution versus magnetic local time}

The density distribution in the plasmasphere varies with the MLT sector considered, and in different ways according to the level of magnetic activity considered (see maps in Plate 1 of Gallagher et al., 2000). During the refilling phase considered in our study, variations with MLT of the density distribution are illustrated in maps of Figs. 5 and 6 and in density plots of Figs. 8 and 9. The main feature, which shows up clearly in all figures, is the day-night asymmetry of the density distribution. It is more important for the class 1 dataset, related to more active conditions, in accordance with views in Gallagher et al. (2000). The density distributions in the noon sector (09:00-15:00 MLT) and in the midnight sector (21:00-03:00 MLT) are qualitatively very different for class 1 data: steeper $n_{\mathrm{e}}(L)$ profile and less measurable densities at high $L$-values in the midnight sector. Differences between day and night distributions are smaller for data of classes 2 and 3 (see Fig. 9). The second clear feature is the dawn-dusk asymmetry, and the gradual increase of median densities with the MLT sector considered (central times from 00:00 to 18:00 MLT). As stated in Sect. 3.2, the average density increase with the refilling duration considered (from one to six days) is sometimes less important (at high $L$-values) than the density increase with the MLT sector (over a $6 \mathrm{~h}$ MLT difference).

The main cause of the MLT dependences described above is the Sun radiation (sunlit ionosphere at the base of magnetic flux tubes in the dayside). This induces a noonmidnight asymmetry on ionosphere-magnetosphere interactions (plasma supplies turning to plasma losses), which becomes also a dawn-dusk asymmetry when considering the co-rotation. Comparing densities at various MLT sectors for a fixed $L$-value could be used to obtain a dayside refilling rate in the frame of the approximation of a stationary convection pattern, because following a given $L$-value means in this case following a single flux tube. However, our datasets are mixing records taken at different durations Dq. Furthermore, the approximation of a stationary convection pattern is not valid. Only qualitative features are meaningful under these conditions.

One interesting qualitative feature is that the density increases from the dawn to the noon sector are significantly smaller than the density increases from the noon to the dusk sector. One explanation could be the presence of drainage plumes, more frequent in the afternoon than in the prenoon sector (Darrouzet et al., 2008). Material eroded from the plasmasphere, forming drainage plumes, are not always lost through the magnetopause boundary, but can stagnate in the afternoon dusk sector (Carpenter et al., 1993), or stroll around in the plasma trough and explain irregularities observed at high $L$-values in Fig. 9. This material could contribute to the refilling process. Another explanation could be the deformation of magnetic field lines (compression in the day, stretching in the night). This induces a noonmidnight asymmetry negligible at low $L$-values, hence often neglected, but important in the high $L$-regions we study here. The realistically modelled magnetic field that we use (Fig. 1) indicates that, at $L=7$, the volume of a magnetic flux tube co-rotating at the equator is significantly smaller on nightside than on dayside, with a midnight versus noon reduction factor of about $40 \%$. This leads to a "breathing" of the co-rotating plasmasphere and its consequences. The change in volume of flux tubes (inflation from dawn to noon, then compression from noon to dusk) might explain why (for a regular total content increase per MLT hour) the density increase is higher from noon to dusk than from dawn to noon. Moreover, and on another line of thought, the plasmasphere breathing likely affects the distribution of particles on trapped-untrapped orbits as well as the convective instability 
situation (since it depends on field line curvature). Plasmasphere breathing might increase the loss of particles at ionospheric level in the night sector, and consequently accentuate the day-night asymmetry due to Sun radiation. We plan in the future to better characterise densities in the night sector in order to analyse this hypothesis.

To conclude this discussion, we think that it is premature to draw a precise, quantitative picture of the 3-D dynamical refilling plasmasphere from our dataset. Nevertheless, several qualitative behaviours are meaningful. These findings provide useful indications toward further developments of 3-D refilling models of the plasmasphere.

\section{Conclusions and perspectives}

The study presented in this paper has been driven by the desire to add data of a new kind to the already large set of observations existing in the plasmasphere region and its surroundings. The WHISPER instrument, which includes a sounder, has been shown to supply reliable density diagnostics (a key parameter for understanding plasma contents and motion) even for regions with no signatures from natural emissions. This is the case in regions of low density (above a lower threshold of $\sim 0.2 \mathrm{~cm}^{-3}$ ) where measurements from particle analysers or spacecraft potential encounter difficulties of various natures. Furthermore, global images from the IMAGE mission, which otherwise have allowed a spectacular progress in understanding the complex nature of plasmasphere morphology and dynamics, could not describe features where densities are below about $40 \mathrm{~cm}^{-3}$ (Goldstein et al., 2003). Another interesting characteristic of WHISPER density dataset is that it covers a large portion of the inner magnetosphere, including regions not explored by previous missions (ISEE 1, GEOS 1 and 2, AKEBONO, DE-1, Viking, POLAR, IMAGE), in particular latitudinal cuts of magnetic flux tubes at high $L$-values. Observations from the WHISPER dataset and its findings can thus be compared and added to the current knowledge formed by the previous in situ, ground-based and remote sensing measurements.

We address one particular topic, the refilling of the plasmasphere during extended quiet periods. The statistical approach used here required a refined magnetic field model, validated via comparison with magnetic field measurements on board CLUSTER. More than three years of Cluster exploration of inner magnetosphere $(2<L<10)$ have been analysed. Comparisons between distributions at three stages of the plasmasphere refilling over the long term (a total time interval of more than six days) show a clear evolution. Several features (increase of the $L$-position of a PBL boundary, general increase of density inside of this boundary, presence of complex structures of cold plasma in the bulge sector) are meeting expectations. The study pinpoints several new findings, mostly in the outermost part of the plasmasphere $(L \geq 6)$. In particular, latitudinal density distributions along field lines point out the presence of a significant density knee at medium latitudes. Above that step, the density distribution varies significantly both with the MLT sector considered and with the refilling duration. Observed characteristics will have to be compared to refilling models adapted to the high $L$ region, where convective instability mechanisms are to be taken into account properly in the context of realistic magnetic field models. Specific plasma sources at high $L$ values, like the auroral fountain (Horwitz and Moore, 1997), and specific wave-particle interactions impacting the refilling mechanism will have to be discussed.

More generally, several perspectives are offered to pursue the exploitation of this WHISPER density dataset. The first step is to increase the dataset, adding densities derived in years 2009 and 2010, when the apex of magnetic flux tubes at high $L$-values has been explored by Cluster. The statistical approach can be improved in several ways. The refilling duration can be better estimated, accounting for the local time position of measurement. Seasonal effects can be studied or taken into account by sorting density records according to the state (sunlit or not) of each of conjugated ionospheric sources. In order to survey the first steps of refilling, the criteria for selecting events will have to be more severe than in this study, and done with more care, by taking into account the initial replenishment of the magnetic flux tubes and by using magnetic indices at a better time resolution.

Acknowledgements. The authors warmly thank both reviewers for their comments, which helped to improve the manuscript. FGM data are retrieved from Cluster Active Archive. The authors thank the technical teams at ESOC, ESTEC, and in LPC2E for their great efforts, leading to acquisition of important scientific databases. CNES and ESA funding supported WHISPER instrument construction and support WHISPER data analysis.

Guest Editor M. Taylor thanks A. Masson and one anonymous referee for their help in evaluating this paper.

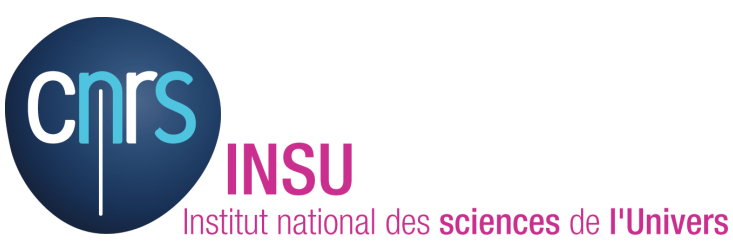

The publication of this article is financed by CNRS-INSU.

\section{References}

André, N. and Lemaire, J. F.: Convective instabilities in the plasmasphere, J. Atmos. Solar-Terr. Phys., 68, 213-227, 2006.

Balogh, A., Dunlop, M. W., Cowley, S. W. H., Southwood, D. J., Thomlinson, J. G., Glassmeier, K. H., Musmann, G., Luhr, H., Buchert, S., Acuna, M. H., Fairfield, D. H., Slavin, J. A., Riedler, W., Schwingenschuh, K., and Kivelson, M. G.: The 
Cluster Magnetic Field Investigation, Space Sci. Rev., 79, 6591, doi:10.1023/A:1004970907748, 1997.

Belmont, G.: Characteristic frequencies of a non-Maxwellian plasma: a method for localizing the exact frequencies of magnetospheric intense natural waves near Fpe, Planet. Space Sci., 29, 1251-1266, 1981.

Bernstein, I. B.: Waves in plasma in a magnetic field, Phys. Review, 109, 10-21, 1958.

Borovsky, J. E. and Denton, M. H.: Electron loss rates from the outer radiation belt caused by the filling of the outer plasmasphere: The calm before the storm, J. Geophys. Res., 114, A11203, doi:10.1029/2009JA014063, 2009.

Borovsky, J. E. and Steinberg, J. T.: The "calm before the storm" in CIR/magnetosphere interactions: Occurrence statistics, solar wind statistics, and magnetospheric preconditioning, J. Geophys. Res., 111, A07S10, doi:10.1029/2005JA011397, 2006.

Canu, P., Décréau, P. M. E., Trotignon, J. G., Rauch, J. L., Seran, H. C., Fergeau, P., Lévêque, M., Martin, Ph., Sené, F. X., Le Guirriec, E., Alleyne, H., and Yearby, K.: Identification of natural plasma emissions observed close to the plasmapause by the Cluster-Whisper relaxation sounder, Ann. Geophys., 19, 16971709, doi:10.5194/angeo-19-1697-2001, 2001.

Carpenter, D. L.: Whistler Evidence of a 'Knee' in the Magnetospheric Ionization Density Profile, J. Geophys. Res., 68, 16751682, doi:10.1029/JZ068i006p01675, 1963.

Carpenter, D. L.: Relations between the dawn minimum in the equatorial radius of the plasmapause and Dst, $\mathrm{Kp}$ and local K at Byrd station, J. Geophys. Res., 72, 2969-2971, doi:10.1029/JZ072i011p02969, 1967.

Carpenter, D. L.: Whistler Evidence of the Dynamic Behavior of the Duskside Bulge in the Plasmasphere, J. Geophys. Res., 75, 3837-3847, doi:10.1029/JA075i019p03837, 1970.

Carpenter, D. L. and Anderson, R. R.: An ISEE/Whistler model of equatorial electron density in the magnetosphere, J. Geophys. Res., 97, 1097-1108, doi:10.1029/91JA01548, 1992.

Carpenter, D. L. and Lemaire, J.: The Plasmasphere Boundary Layer, Ann. Geophys., 22, 4291-4298, doi:10.5194/angeo-224291-2004, 2004.

Carpenter, D. L., Giles, B. L., Chappell, C. R., Décréau, P. M. E., Anderson, R. R., Persoon, A. M., Smith, A. J., Corcuff, Y., and Canu, P.: Plasmasphere dynamics in the duskside bulge region: A new look at old topic, J. Geophys. Res., 98, 19243-19271, doi:10.1029/93JA00922, 1993.

Chandler, M. O. and Moore, T. E.: Observations of the geopause at the equatorial magnetopause: Density and temperature, Geophys. Res. Lett., 30, 1869, doi:10.1029/2003GL017611, 2003.

Chappell, C. R.: Recent satellite measurements of the morphology and dynamics of the plasmasphere., Rev. Geophys. Space Phys., 10, 951-979, 1972.

Chappell, C. R., Harris, K. K., and Sharp, G. W.: A study of the influence of magnetic activity on the location of the plasmapause as measured by OGO 5., J. Geophys. Res., 75, 50-56, doi:10.1029/JA075i001p00050, 1970.

Chasovitin, Y. K., Gulyaeva, T. L., Deminov, M. G., and Ivanova, S. E.: Russian standard model of Geophys ionosphere (SMI), Preprint No.1(1110), IZMIRAN, Moscow, Russia, 19 pp., Proc. of COST 251 Workshop, Side, Turkey, COST251TD(98)005, RAL, UK, pp. 161-172, 1998.
Corcuff, P., Corcuff, Y., Carpenter, D. L., Chappell, C. R., Vigneron, J., and Kleimenova, N.: La plasmasphère en période de recouvrement magnétique: Etude combinée des données des satellites OGO 4, OGO 5 et des sifflements reçus au sol, Ann. Geophys., 28, 679-696, 1972.

Dandouras, I.: Detection of plasmaspheric wind by the Cluster spacecraft, and contribution to the magnetospheric populations, Geophys. Res. Abs., 11, 5360, 2008.

Darrouzet, F., De Keyser, J., Décréau, P. M. E., Lemaire, J. F., and Dunlop, M. W.: Spatial gradients in the plasmasphere from Cluster, Geophys. Res. Lett., 330, L08105, doi:10.1029/2006GL025727, 2006.

Darrouzet, F., De Keyser, J., Décréau, P. M. E., El LemdaniMazouz, F., and Vallières, X.: Statistical analysis of plasmaspheric plumes with Cluster/WHISPER observations, Ann. Geophys., 26, 2403-2417, doi:10.5194/angeo-26-2403-2008, 2008.

Darrouzet, F., de Keyser, J., and Pierrard, V. (Eds.): The Earth's Plasmasphere: A Cluster and Image perspective, 296 pp., Springer, vol. 145, doi:10.1007/978-1-4419-1323-4, 2009a.

Darrouzet, F., Gallagher, D. L., André, N., Carpenter, D. L., Dandouras, I., Décréau, P. M. E., de Keyser, J., Denton, R. E., Foster, J. C., Goldstein, J., Moldwin, M. B., Reinisch, B. W., Sandel, B. R., and Tu, J.: Plasmaspheric Density Structures and Dynamics: Properties Observed by the CLUSTER and IMAGE Missions, Space Sci. Rev., 145, 55-106, doi:10.1007/s11214-0089438-9, 2009b.

De Keyser, J., Roth, M., and Lemaire, J.: The magnetospheric driver of subauroral ion drifts, Geophys. Res. Lett., 25, 1625-1628, doi:10.1029/98GL01135, 1998.

Décréau, P. M. E., Béghin, C., and Parrot, M.: Global characteristics of the cold plasma in the equatorial plasmapause region, as deduced from the GEOS 1 Mutual Impedance Experiment, J. Geophys. Res., 87, 695-712, doi:10.1029/JA087iA02p00695, 1982.

Décréau, P. M. E., Fergeau, P., Krannosels'kikh, V., Lévêque, M., Martin, P., Randriamboarison, O., Sene, F. X., Trotignon, J. G., Canu, P., and Mögensen, P. B.: Whisper, a Resonance Sounder and Wave Analyser: Performances and Perspectives for the Cluster Mission, Space Sci. Rev., 79, 157-193, doi:10.1023/A:1004931326404, 1997.

Décréau, P. M. E., Fergeau, P., Krasnoselskikh, V., Le Guirriec, E., Lévêque, M., Martin, Ph., Randriamboarison, O., Rauch, J. L., Sené, F. X., Séran, H. C., Trotignon, J. G., Canu, P., Cornilleau, N., de Féraudy, H., Alleyne, H., Yearby, K., Mögensen, P. B., Gustafsson, G., André, M., Gurnett, D. C., Darrouzet, F., Lemaire, J., Harvey, C. C., Travnicek, P., and Whisper experimenters: Early results from the Whisper instrument on Cluster: an overview, Ann. Geophys., 19, 1241-1258, doi:10.5194/angeo-19-1241-2001, 2001.

Denton, R. E., Goldstein, J., and Menietti, J. D.: Field line dependence of magnetospheric electron density, Geophys. Res. Lett., 29, 2205-2209, doi:10.1029/2002GL015963, 2002a.

Denton, R. E., Goldstein, J., Menietti, J. D., and Young, S. L.: Magnetospheric electron density model inferred from Polar plasma wave data, J. Geophys. Res., 107, 1386, doi:10.1029/2001JA009136, 2002b.

Denton, R. E., Menietti, J. D., Goldstein, J., Young, S. L., and Anderson, R. R.: Electron density in the magnetosphere, J. Geophys. Res., 109, A09215, doi:10.1029/2003JA010245, 2004. 
Denton, R. E., Décréau, P., Engebretson, M. J., Darrouzet, F., Posch, J. L., Mouikis, C., Kistler, L. M., Cattell, C. A., Takahashi, K., Schäfer, S., and Goldstein, J.: Field line distribution of density at $L=4.8$ inferred from observations by CLUSTER, Ann. Geophys., 27, 705-724, doi:10.5194/angeo-27-705-2009, 2009.

Elphic, R. C., Thomsen, M. F., and Borovsky, J. E.: The fate of the outer plasmasphere, Geophys. Res. Lett., 24, 365-368, doi:10.1029/97GL00141, 1997.

Escoubet, C. P., Schmidt, R., and Goldstein, M. L.: Cluster Science and Mission Overview, Space Sci. Rev., 79, 11-32, doi:10.1023/A:1004923124586, 1997.

Escoubet, C. P., Fehringer, M., and Goldstein, M.: Introduction: The Cluster mission, Ann. Geophys., 19, 1197-1200, doi:10.5194/angeo-19-1197-2001, 2001.

Finlay, C. C., Maus, S., Beggan, C. D., Bondar, T. N., Chambodut, A., Chernova, T. A., Chulliat, A., Golovkov, V. P., Hamilton, B., Hamoudi, M., Holme, R., Hulot, G., Kuang, W., Langlais, B., Lesur, V., Lowes, F. J., Lühr, H., Macmillan, S., Mandea, M., McLean, S., Manoj, C., Menvielle, M., Michaelis, I., Olsen, N., Rauberg, J., Rother, M., Sabaka, T. J., Tangborn, A., TøffnerClausen, L., Thébault, E., Thomson, A. W. P., Wardinski, I., Wei, Z., and Zvereva, T. I.: International Geomagnetic Reference Field: the eleventh generation, Geophys. J. Inter., 183, 12161230, doi:10.1111/j.1365-246X.2010.04804.x, 2010.

Gallagher, D. L., Craven, P. D., and Comfort, R. H.: Global core plasma model, J. Geophys. Res., 105, 18819-18834, doi:10.1029/1999JA000241, 2000.

Goldstein, J., Spasojević, M., Reiff, P. H., Sandel, B. R., Forrester, W. T., Gallagher, D. L., and Reinisch, B. W.: Identifying the plasmapause in IMAGE EUV data using IMAGE RPI in situ steep density gradients, J. Geophys. Res., 108, 1147, doi:10.1029/2002JA009475, 2003.

Goldstein, J., Sandel, B. R., Thomsen, M. F., Spasojević, M., and Reiff, P. H.: Simultaneous remote sensing and in situ observations of plasmaspheric drainage plumes, J. Geophys. Res., 109, A03202, doi:10.1029/2003JA010281, 2004.

Higel, B. and Lei, W.: Electron density and plasmapause characteristics at 6.6 earth radii - A statistical study of the GEOS 2 relaxation sounder data, J. Geophys. Res., 89, 1583-1601, doi:10.1029/JA089iA03p01583, 1984.

Horwitz, J. L. and Moore, T. E.: Four contemplorary issues concerning ionospheric plasma flow to the magnetosphere, Space Sci. Rev., 80, 49-76, doi:10.1023/A:1004973603955, 1997.

Horwitz, J. L., Comfort, R. H., and Chappell, C. R.: A statistical characterization of plasmasphere density structure and boundary locations, J. Geophys. Res., 95, 7937-7947, doi:10.1029/JA095iA06p07937, 1990.

Huang, X., Reinisch, B. W., Song, P., Green, J. L., and Gallagher, D. L.: Developing an empirical density model of the plasmasphere using IMAGE/RPI observations, Adv. Space Res., 33, 829-832, doi:10.1016/j.asr.2003.07.007, 2004.

Johnstone, A. D., Alsop, C., Burge, S., Carter, P. J., Coates, A. J., Coker, A. J., Fazakerley, A. N., Grande, M., Gowen, R. A., Gurgiolo, C., Hancock, B. K., Narheim, B., Preece, A., Sheather, P. H., Winningham, J. D., and Woodliffe, R. D.: Peace: a Plasma Electron and Current Experiment, Space Sci. Rev., 79, 351-398, doi:10.1023/A:1004938001388, 1997.

Kougblénou, S., Lointier, G., Décréau, P. M. E., Trotignon, J.-G., Rauch, J.-L., Vallières, X., Canu, P., Masson, A., and Pickett,
J.: Lower hybrid resonances stimulated by the four CLUSTER relaxation sounders deep inside the plasmasphere: observations and inferred plasma characteristics, Ann. Geophys., 29, 2003 2018, doi:10.5194/angeo-29-2003-2011, 2011.

Laakso, H., Pfaff, R., and Janhunen, P.: Polar observations of electron density distribution in the Earth's magnetosphere. 2. Density profiles, Ann. Geophys., 20, 1725-1735, doi:10.5194/angeo-201725-2002, 2002.

Lam, M. M., Horne, R. B., Meredith, N. P., and Glauert, S. A.: Modeling the effects of radial diffusion and plasmaspheric hiss on outer radiation belt electrons, Geophys. Res. Lett., 342, L20112, doi:10.1029/2007GL031598, 2007.

Larsen, B. A., Klumpar, D. M., and Gurgiolo, C.: Correlation between plasmapause position and solar wind parameters, J. Atmos. Solar-Terr. Phys., 69, 334-340, doi:10.1016/j.jastp.2006.06.017, 2007.

Lemaire, J.: The 'Roche-limit' of ionospheric plasma and the formation of the plasmapause, Planet. Space Sci., 22, 757-766, 1974.

Lemaire, J. F. and Gringauz, K. I.: The Earth's Plasmasphere, Cambridge, UK: Cambridge University Press, 350 pp., 1998.

Lemaire, J. and Schunk, R. W.: Plasmaspheric wind, J. Atmos. Solar-Terr. Phys., 54, 467-477, 1992.

Liemohn, M. W., Khazanov, G. V., and Kozyra, J. U.: Guided plasmaspheric hiss interactions with superthermal electrons 1 . Resonance curves and timescales, J. Geophys. Res., 1021, 1161911624, doi:10.1029/97JA00825, 1997.

Maynard, N. C. and Grebowsky, J. M.: The plasmapause revisited, J. Geophys. Res., 82, 1591-1600, doi:10.1029/JA082i010p01591, 1977.

Mayaud, P. N.: Derivation, Meaning and Use of Geomagnetic Indices, Am. Geophys. Union, Washington D.C., Geophysical Monograph 22, 154 pp., doi:10.1029/GM022, 1980.

McFadden, J. P., Carlson, C. W., Larson, D., Bonnell, J., Mozer, F. S., Angelopoulos, V., Glassmeier, K.-H., and Auster, U.: Structure of plasmaspheric plumes and their participation in magnetopause reconnection: First results from THEMIS, Geophys. Res Lett., 351, L17S10, doi:10.1029/2008GL033677, 2008.

Menvielle, M. and Berthelier, A.: The K-derived planetary indices: Description and availability, Rev. Geophys., 29, 415-432, doi:10.1029/91RG00994, 1991

Moldwin, M. B., Thomsen, M. F., Bame, S. J., McComas, D. J., and Moore, K. R.: An examination of the structure and dynamics of the outer plasmasphere using multiple geosynchronous satellites, J. Geophys. Res., 99, 11475-11482, doi:10.1029/93JA03526, 1994.

Moldwin, M. B., Thomsen, M. F., Reeves, G. D., and McComas, D. J.: The dynamic plasmasphere, Adv. Space Res., 20, 395-400, doi:10.1016/S0273-1177(97)00699-6, 1997.

Moldwin, M. B., Downward, L., Rassoul, H. K., Carpenter, D. L., and Anderson, R. R.: A New Model of the Location of the Plasmapause: CRRES Results, J. Geophys. Res., 107, 1339, doi:10.1029/2001JA009211, 2002.

Nishida, A.: Formation of Plasmapause, or Magnetospheric Plasma Knee, by the Combined Action of Magnetospheric Convection and Plasma Escape from the Tail, J. Geophys. Res., 71, 5669, 1966.

Persoon, A. M., Gurnett, D. A., Peterson, W. K., Waite, Jr., J. H., Burch, J. L., and Green, J. L.: Electron density depletions in 
the nightside auroral zone, J. Geophys. Res., 93, 1871-1895, doi:10.1029/JA093iA03p01871, 1988.

Pierrard, V. and Cabrera, J.: Comparisons between EUV/IMAGE observations and numerical simulations of the plasmapause formation, Ann. Geophys., 23, 2635-2646, doi:10.5194/angeo-232635-2005, 2005.

Pierrard, V. and Stegen, K.: A three-dimensional dynamic kinetic model of the plasmasphere, J. Geophys. Res., 113, A10209, doi:10.1029/2008JA013060, 2008.

Reinisch, B. W., Huang, X., Song, P., Sales, G. S., Fung, S. F., Green, J. L., Gallagher, D. L., and Vasyliunas, V. M.: Plasma Density Distribution Along the Magnetospheric Field: RPI Observations From IMAGE, Geophys. Res. Lett., 28, 4521-4524, doi:10.1029/2001GL013684, 2001.

Reinisch, B. W., Huang, X., Song, P., Green, J. L., Fung, S. F., Vasyliunas, V. M., Gallagher, D. L., and Sandel, B. R.: Plasmaspheric mass loss and refilling as a result of a magnetic storm, J. Geophys. Res., 109, A01202, doi:10.1029/2003JA009948, 2004.

Reinisch, B. W., Moldwin, M. B., Denton, R. E., Gallagher, D. L., Matsui, H., Pierrard, V., and Tu, J.: Augmented Empirical Models of Plasmaspheric Density and Electric Field Using IMAGE and CLUSTER Data, Space Sci. Rev., 145, 231-261, doi:10.1007/s11214-008-9481-6, 2009.

Reynolds, M. A., Ganguli, G., Su, Y.-J., and Thomsen, M. F.: The local-time variation of the quiet plasmasphere: geosynchronous observations and kinetic theory, Ann. Geophys., 21, 2147-2154, doi:10.5194/angeo-21-2147-2003, 2003.

Sheeley, B. W., Moldwin, M. B., Rassoul, H. K., and Anderson, R. R.: An empirical plasmasphere and trough density model: CRRES observations, J. Geophys. Res., 106, 25631-25642, doi:10.1029/2000JA000286, 2001.

Singh, N. and Horwitz, J. L.: Plasmaspheric refilling: recent observations and modeling, J. Geophys. Res., 97, 1049-1079, doi:10.1029/91JA02602, 1992.

Spasojević, M., Goldstein, J., Carpenter, D. L., Inan, U. S., Sandel, B. R., Moldwin, M. B., and Reinisch, B. W.: Global response of the plasmasphere to a geomagnetic disturbance, J. Geophys. Res., 108, 1340, doi:10.1029/2003JA009987, 2003.

Spasojević, M., Frey, H. U., Thomsen, M. F., Fuselier, S. A., Gary, S. P., Sandel, B. R., and Inan, U. S.: The link between a detached subauroral proton arc and a plasmaspheric plume, Geophys. Res. Lett., 31, L04803, doi:10.1029/2003GL018389, 2004.

Summers, D. and Thorne, R. M.: Relativistic electron pitchangle scattering by electromagnetic ion cyclotron waves during geomagnetic storms, J. Geophys. Res., 108, 1143, doi:10.1029/2002JA009489, 2003.

Summers, D., Ma, C., and Mukai, T.: Competition between acceleration and loss mechanisms of relativistic electrons during geomagnetic storms, J. Geophys. Res., 109, A04221, doi:10.1029/2004JA010437, 2004.
Trotignon, J. G., Etcheto, J., and Thouvenin, J. P.: Automatic determination of the electron density measured by the relaxation sounder on board ISEE 1, J. Geophys. Res., 91, 4302-4320, doi:10.1029/JA091iA04p04302, 1986.

Trotignon, J. G., Décréau, P. M. E., Rauch, J. L., Vallières, X., Rochel, A., Kougblénou, S., Lointier, G., Facskó, G., Canu, P., Darrouzet, F., and Masson, A.: The WHISPER Relaxation Sounder and the CLUSTER Active Archive, Springer, Part 1, 185-208, doi:10.1007/978-90-481-3499-1_12, 2010.

Tsyganenko, N. A. and Sitnov, M. I.: Modeling the dynamics of the inner magnetosphere during strong geomagnetic storms, J. Geophys. Res., 110, A03208, doi:10.1029/2004JA010798, 2005.

Tu, J.-N., Horwitz, J. L., Nsumei, P. A., Song, P., Huang, X.-Q., and Reinisch, B. W.: Simulation of polar cap field-aligned electron density profiles measured with the IMAGE radio plasma imager, J. Geophys. Res., 109, A07206, doi:10.1029/2003JA010310, 2004.

Tu, J., Song, P., Reinisch, B. W., Huang, X., Green, J. L., Frey, H. U., and Reiff, P. H.: Electron density images of the middleand high-latitude magnetosphere in response to the solar wind, J. Geophys. Res., 110, A12210, doi:10.1029/2005JA011328, 2005.

Tu, J., Song, P., Reinisch, B. W., Green, J. L., and Huang, X.: Empirical specification of field-aligned plasma density profiles for plasmasphere refilling, J. Geophys. Res., 111, A06216, doi:10.1029/2005JA011582, 2006.

Tu, J., Song, P., Reinisch, B. W., and Green, J. L.: Smooth electron density transition from plasmasphere to the subauroral region, J. Geophys. Res., 112, A05227, doi:10.1029/2007JA012298, 2007.

Usanova, M. E., Mann, I. R., Kale, Z. C., Rae, I. J., Sydora, R. D., Sandanger, M., Søraas, F., Glassmeier, K.-H., Fornacon, K.-H., Matsui, H., Puhl-Quinn, P. A., Masson, A., and Vallières, X.: Conjugate ground and multisatellite observations of compression-related EMIC Pc1 waves and associated proton precipitation, J. Geophys. Res., 115, A07208, doi:10.1029/2009JA014935, 2010.

Wilson, G. R., Horwitz, J. L., and Lin, J.: A Semikinetic Model for Early Stage Plasmasphere Refilling, 1, Effects of Coulomb Collisions, J. Geophys. Res, 97, 1109-1119, doi:10.1016/02731177(93)90320-B, 1992.

Woodfield, E. E., Dunlop, M. W., Holme, R., Davies, J. A., and Hapgood, M. A.: A comparison of Cluster magnetic data with the Tsyganenko 2001 model, J. Geophys. Res., 112, 6248, doi:10.1029/2006JA012217, 2007.

Zhang, Q.-H., Dunlop, M. W., Holme, R., and Woodfield, E. E.: Comparison of eight years magnetic field data from Cluster with Tsyganenko models in the inner magnetosphere, Ann. Geophys., 28, 309-326, doi:10.5194/angeo-28-309-2010, 2010. 